\title{
Feeling textures through a probe: Effects of probe and surface geometry and exploratory factors
}

\author{
ROBERTA L. KLATZKY \\ Carnegie Mellon University, Pittsburgh, Pennsylvania \\ SUSAN J. LEDERMAN, CHERYL HAMILTON, and MOLLY GRINDLEY \\ Queen's University, Kingston, Ontario, Canada \\ and \\ ROBERT H. SWENDSEN \\ Carnegie Mellon University, Pittsburgh, Pennsylvania
}

\begin{abstract}
Vibratory roughness perception occurs when people feel a surface with a rigid probe. Accordingly, perceived roughness should reflect probe and surface geometry, exploratory speed, and force. Experiments 1 and 2 compared magnitude estimation of roughness with the bare finger and two types of probes, one designed to eliminate force moments, under the subject's active control. Experiments 3 and 4 varied speed under passive control. Log magnitude was consistently a quadratic function of log spacing between elements in the surface. The location of the function's peak was related to the drop pointthat is, the spacing at which the probe can just drop between elements-which is affected by probe tip diameter, element height, and speed. Other parameters of the quadratic were affected by probe type and speed.
\end{abstract}

Feeling with a probe may seem unusual, but in fact, it is commonplace. For example, when people scrape the bottom of a pot with a spoon or write with a pencil on grained paper, the perceptual impression of a textured surface arises from the vibrations transmitted to the fingers that hold the implement. As Katz (1925/1989) noted, people have a rich impression of the surface, not of the vibrations themselves.

A theoretical account of this perceptual process must take into account three general components: (1) the physics of the probe-tip/surface interaction and the transmission of vibrations through the probe shaft, (2) the filtering imposed by the skin and the responses of the mechanoreceptors, and (3) higher order factors that might alter roughness perception, such as active versus passive control of exploration and, correspondingly, the role of efferent commands. In the present experiments, we examined the effects of variables that are related to the first of these influences. The manipulated variables are of two types. One pertains to the geometric properties of the probe and the textured surface, including the

This research was partially supported by the National Science and Engineering Research Council of Canada. Data from Experiment 3 were previously reported in Lederman, Klatzky, Hamilton, and Grindley (2000) and were adapted with permission for Figure 6. We thank Robert Howe for suggesting the zero-moment probe. We also thank Jesse Kates and Andrew Thompson for assistance with data collection and analysis. Correspondence concerning this article should be addressed to R. L. Klatzky, Department of Psychology, Carnegie Mellon University, Pittsburgh, PA 15213 (e-mail: klatzky@cmu.edu). diameter and shape of the probe and the spacing and shape of the elements that form the surface. The other pertains to the nature of exploration, including the speed with which the probe is passed over the plate and the posture of the hand holding the probe. Before considering the potential effects of these factors, we first will review theories of direct texture perception with the bare finger.

\section{Texture Perception Via the Bare Finger}

A substantial amount of research on haptic perception has been directed at understanding how people perceive the property of roughness as they explore a textured surface with the bare skin. The model that has resulted from this research was characterized by Klatzky and Lederman (1999) as spatial intensive in nature, because it assumes that processing begins with a representation of the surface as a spatial pressure map and culminates with a unidimensional estimate of roughness magnitude.

This model of roughness perception is based on studies that systematically manipulated the geometric properties of textured surfaces and the nature of exploration, while measuring behavioral and neurophysiological responses. Psychophysical research has led to an understanding of how texture perception varies with the interaction between skin and surface, whereas neurophysiological research has indicated the underlying peripheral and central neural computations. Using psychophysical methods, Taylor and Lederman (1975) found that the perceived roughness of a surface was directly related to the total area of skin that was instantaneously deformed from a baseline resting position while in contact with a surface 
(a linear grating). The skin area and, accordingly, the roughness percept were affected by surface variables, such as groove width and ridge width (the former accounting for considerably greater variance in roughness judgments than the latter), and by exploratory parameters, such as force (Lederman, 1974). Johnson and associates (see Johnson \& Hsiao, 1994) developed a neurophysiological coding model to describe the processing from skin deformation to roughness percept. According to the model, a spatial map of intensity values is provided by slowly adapting Type I peripheral mechanoreceptors. Signals passed to the primary somatosenory area in the cortex (area $3 \mathrm{~b}$ of SI) are processed by units with excitatory and inhibitory subregions, which compute differences in the mechanoreceptor activity. At a subsequent stage, the differences are integrated in cortical area SII. At this point, information about the spatial layout of the input is no longer preserved; rather, the output is a signal corresponding to the overall roughness magnitude.

The model of roughness perception via skin contact has not implicated vibratory coding, when the elements forming the texture are separated by $1 \mathrm{~mm}$ or more (macrotextures). The lack of vibratory influence is indicated by several findings: Relative to the effects of geometric variables and exploratory force, exploration speed has typically produced a small effect on roughness (Lederman, 1974, 1983), as has the spatial period of the elements (Lederman \& Taylor, 1972). Both of these variables should be reflected in vibratory frequency. A direct test of the vibratory model by Lederman, Loomis, and Williams (1982) showed that roughness judgments were unaffected by adapting the fingertip to selective vibrotactile inputs ( 20 or $250 \mathrm{~Hz}$; but see Kudoh, 1988, for a small and variable effect).

Smith, Chapman, Deslandes, Langlais, and Thibodeau (2002) recently proposed, however, that a temporal model of roughness is needed in addition to a spatial one. Using raised-dot elements that formed tight columns and varying the spacing between them, they found that roughness can be predicted by the root-mean square of the tangential forces that occur as people stroke a plate. They suggested that temporal variations in the amplitude of skin displacement might provide a roughness code. Neural recordings (Birznieks, Jenmalm, Goodwin, \& Johansson, 2001) suggest that both rapidly and slowly adapting mechanoreceptors that respond to tangential forces might underlie this type of code.

The role of vibration is clearly implicated for surfaces with elements spaced more densely than $1 \mathrm{~mm}$ (microtextures; see Hollins, Bensmaia, \& Risner, 1998; LaMotte $\&$ Srinivasan, 1991). However, even with these finegrained surfaces, spatial coding is also indicated. Using gratings having groove widths as small as $0.1 \mathrm{~mm}$, Yoshioka, Gibb, Dorsch, Hsiao, and Johnson (2001) reported that the activity of slowly adapting mechanoreceptors, which perform spatial coding, was correlated with roughness perception. Using particulate textures, Hollins and
Risner (2000) found evidence for a transition between vibratory and spatial coding at a similar particle size.

\section{Texture Perception Via a Probe}

The role of vibratory texture coding is markedly different when we consider exploration of a texture with a probe, rather than with the bare skin. The probe provides a rigid link between the skin and the surface. Direct surface contact by the bare finger produces a spatial map of activation in slowly adapting mechanoreceptors that is directly correlated with the features of the surface. But when the finger holds a probe that contacts the surface, the spatial map reflects the contours of the probe, not those of the surface. The surface properties that constitute texture now give rise to vibrations, which are transmitted to the skin along the rigid link.

How well do people perceive the roughness of a surface by exploring it with a rigid probe? This question was asked by Klatzky, Lederman, and associates (Klatzky \& Lederman, 1999; Lederman, Klatzky, Hamilton, \& Grindley, 2000; Lederman, Klatzky, Hamilton, \& Ramsay, 1999) in a series of studies. Subjects performed magnitude estimation and roughness comparison tasks while feeling a texture with a stylus-shaped probe. Perceived roughness was found to vary systematically with the surface geometry - that is, the spacing between raised elements. However, the nature of the roughness variation with spacing was different with a probe from that found with the bare finger. Magnitude estimation with the bare finger typically gives rise to a linear function relating $\log$ magnitude to log spacing, up to a spacing level at which the elements are so sparse that the surface does not feel textured (but cf. Smith et al., 2002). These studies showed that when the same surfaces were explored with a rigid probe that had a tip small enough to fall between the elements at some spacing value in the manipulated range, the magnitude estimation function (log magnitude against log spacing) was better fit by a quadratic trend.

Furthermore, Klatzky and Lederman (1999) found that the spacing value at which the function peaked was close to the contact area of the probe tip. This suggested that the roughness percept reached a maximum when the geometry of the probe/surface interaction caused a qualitative shift in the nature of contact. That is, near the point at which the average spacing between elements exceeds the probe tip diameter, there should be a transition from the probe's riding on the upper surfaces of the elements to riding on the substrate below. The authors proposed that roughness magnitude might reach a maximum at this transition, which we call the drop point, due to peaks in the vibratory frequency and/or amplitude. The drop point is affected not only by the spacing, but also by the probe tip diameter, the height of the surface elements, and speed, as we will explain below.

However, at best, the experiments of Klatzky and Lederman (1999) could point only to the probe/surface geometry and its effects on vibration as the potential cause of the quadratic trend in the roughness magnitude 
function. In that research, only one probe tip diameter, $2 \mathrm{~mm}$, was small enough to fall within the range of interelement spacing values used in the experiments. A larger probe diameter, $8 \mathrm{~mm}$, gave rise to a more linear function that was similar to the one obtained with the bare finger. The similarity presumably occurred because in neither case was there a spacing value that permitted the exploring effector to fall between the elements constituting the surface.

The effects of exploration speed on roughness perception with a probe also prove to be complex. Lederman et al. (1999) manipulated speed over approximately a 10 -fold range, under active and passive exploration. The peak of the magnitude estimation function occurred at a higher spacing for higher speeds. This outcome is consistent with the idea that the location of the peak is related to the drop point, which would be expected to shift upward on the spacing axis with higher speed, owing to the reduced time allowed for the probe to drop between elements. Finally, speed and spacing exhibited an interaction. At low spacing values, roughness magnitude estimation decreased with increasing speed, but as the elements became more widely spaced, the magnitude estimation functions converged, ultimately crossing over at the highest spacing. We will consider implications of this pattern in the next section.

\section{ANALYSES OF TEXTURE/PROBE/SKIN INTERACTIONS}

In this section, we will present three analyses of physical variables that affect the interactions between a surface texture, a probe, and the skin in contact with the probe. The first is a simplified model that predicts the drop point from the geometry of the probe and the surface, without taking into account the effects of movement during exploration. In the second analysis, we will consider how geometric variables in conjunction with exploratory variables might affect perceived roughness, through the mediating variables of vibratory amplitude and frequency. In the third analysis, we will consider how the posture with which a probe is held will affect the transmission of vibratory information to the skin.

\section{Predicting the Drop Point From Geometric Variables}

This analysis predicts the drop point - the value of spacing at which the probe just penetrates between the elements on a textured surface-from the variables of probe tip diameter, spacing of the elements, and height and shape of the elements (using the two shapes used in the present experiments). We will consider a simplified two-dimensional surface with regular spacing between elements and static probing - that is, without consideration of movement speed or force.

Figure 1A schematizes the geometric interaction when cylindrical raised elements form the textures on plates (Experiments 1 and 2 of this paper). Depth of pen- etration is defined as the distance between the top of the element and the bottom of the probe tip, which reaches a maximum value equal to the height of the element. When the tip has not penetrated to the floor of the plate (i.e., $d<h$ in the figure), the depth of penetration $(d)$ depends on the radius of the probe tip $(r)$ and the interelement spacing $(s)$, by the following equation:

$$
d=r-\sqrt{r^{2}-\left(\frac{s}{2}\right)^{2}} .
$$

As long as the probe diameter is equal to or larger than the interelement spacing, the depth of penetration increases with spacing until it penetrates to the full height of the element (i.e., when $r \geq s / 2, d$ increases with $s$ until $d=h)$. When the probe diameter is smaller than the interelement spacing, it will fall to the floor of the plate and will always penetrate to the height of the element.

Two experiments here ( 3 and 4 ) used truncated-coneshaped elements. The geometric analysis of the point at which the probe could maximally penetrate between the elements is shown in Figures $1 \mathrm{~B}$ and $1 \mathrm{C}$. When the probe is large relative to the interelement spacing (1B), the geometry is the same as for cylindrical cones, because the probe makes contact at the top of the element. With smaller probes, the tip comes to rest against the side of the conical element when penetration is maximal. The equation for that case is shown in Figure 1C.

\section{Effects of Geometric and Exploratory Variables on Perceived Roughness Via Vibration}

The second analysis is concerned with how geometric and exploratory variables would affect roughness magnitude, as mediated by their effects on the vibratory profile. Our fundamental assumption is that the perception of roughness via exploration with a probe is mediated by the amplitude and frequency of the vibration that reaches the skin. These vibratory parameters depend on probe/plate geometry and exploration. We will focus here on the geometric variable of interelement spacing and the exploratory variable of speed. Figure 2 provides a set of initial hypotheses about the nature of this dependence. The proposed mechanisms are speculative but in qualitative accord with existing data on spacing and speed effects. These effects are outlined in three steps: The first step is to suggest how spacing and speed might affect vibratory amplitude and frequency; the second step is to suggest how amplitude and frequency might affect perceived roughness; and from this follows, third, the resultant effects of spacing and speed on perceived roughness.

As is shown in the figure, we propose that the drop point corresponds to a critical transition in the effects of speed and interelement spacing on vibratory amplitude and frequency. Consider first the effects on amplitude (top left panel): For spacing values below the drop point, the depth to which the probe tip penetrates the elements should increase as the spacing increases but should decrease as the speed increases. This means that at dense spacings, the amplitude of vibration will increase with 
(A)
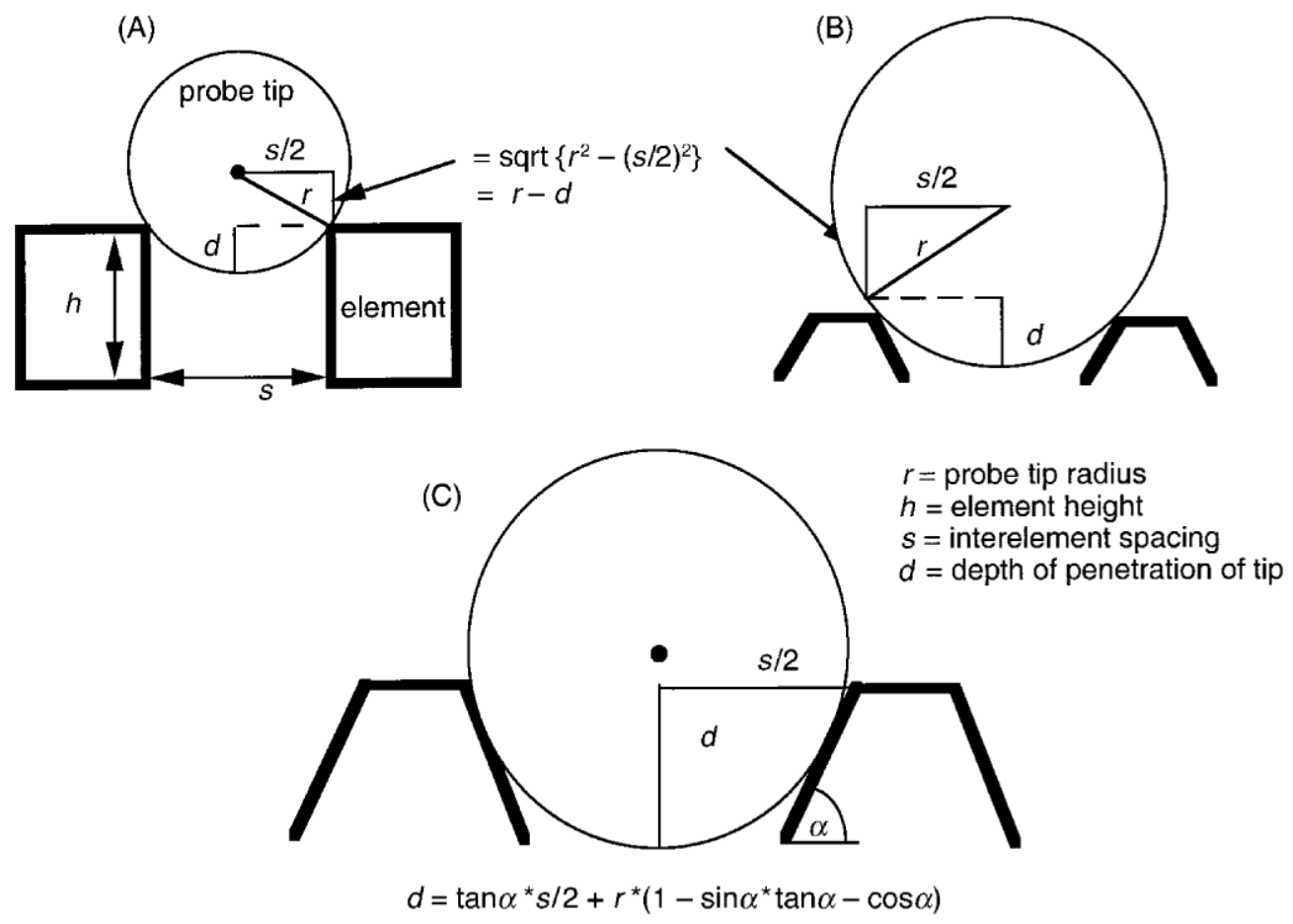

Figure 1. Geometric analysis of the interaction between probe tip and raised elements on a textured surface. The depth of penetration of the probe tip is determined as a function of element height, tip radius, and interelement spacing. The critical value of spacing is that at which $d$ reaches the maximum value (i.e., $d=h$ ). (A) Cylindrical elements (Experiments 1 and 2); (B) elements shaped like truncated cones (Experiments 3 and 4 ) when the probe tip is large; (C) elements shaped like truncated cones when the probe tip is small and at maximum penetration and the tip lies tangent to the ramp of the element.

spacing and decrease with speed. But beyond the drop point, the probe will penetrate fully between the elements at any spacing and speed, and amplitude should not be systematically affected in the same way. (Figure 2 simply depicts constant amplitude beyond the drop point.) Next, consider the effects of spacing and speed on the frequency of vibration (top right panel). Whereas effects on vibratory amplitude are likely to differ before and after the drop point, the frequency should be similarly affected by geometric and exploratory factors across the full range of spacing. Specifically, the fundamental frequency of vibration should be directly related to the number of elements encountered in a unit of time. This would be determined by the speed divided by the total width of a single element and a single gap (i.e., one spatial period). Note, then, that the crossover interaction between spacing and speed observed by Lederman et al. (1999) implies that roughness magnitude is not governed directly by the fundamental frequency of vibration, which should increase with speed regardless of spacing. In the next paragraph, we will suggest a more complex relation between roughness and vibratory frequency.

Figure 2 (middle panel) presents an initial hypothesis about how the perception of roughness might depend on vibratory amplitude and frequency. This hypothesis is based on studies of suprathreshold vibration perception, under the assumption that the perception of vibration and roughness follow similar trends-admittedly, a speculation. A number of experiments have indicated a monotonic relation between subjective vibration magnitude and amplitude of vibration on the skin (Franzén, 1966; Lederman et al., 1982; Sherrick, 1960; Stevens, 1957; Verrillo, Fraioli, \& Smith, 1969). Thus, if perceived roughness follows the course of perceived vibration, it should increase with amplitude (middle left panel). However, the relationship of subjective vibratory magnitude to frequency is not necessarily monotonic. Verrillo et al. (1969) reported that as frequency increased over the range of $10 \mathrm{~Hz}-1 \mathrm{kHz}$, the displacement necessary to produce equal sensation levels first decreased and then leveled off or increased. Their data indicated that perceived intensity of vibration was positively related to frequency primarily in the low-frequency range and that, depending on the displacement, the sensation/frequency relation could take an inverted-U-shaped form across the frequency range. If roughness and vibration perception follow similar trends, this means that roughness could first increase and then decrease with increases in frequency (middle right panel). In order to account for trends in our previous studies, this shift in the roughness/ frequency relation is further assumed to occur around the drop point. 
AMPLITUDE X SPACING \& SPEED
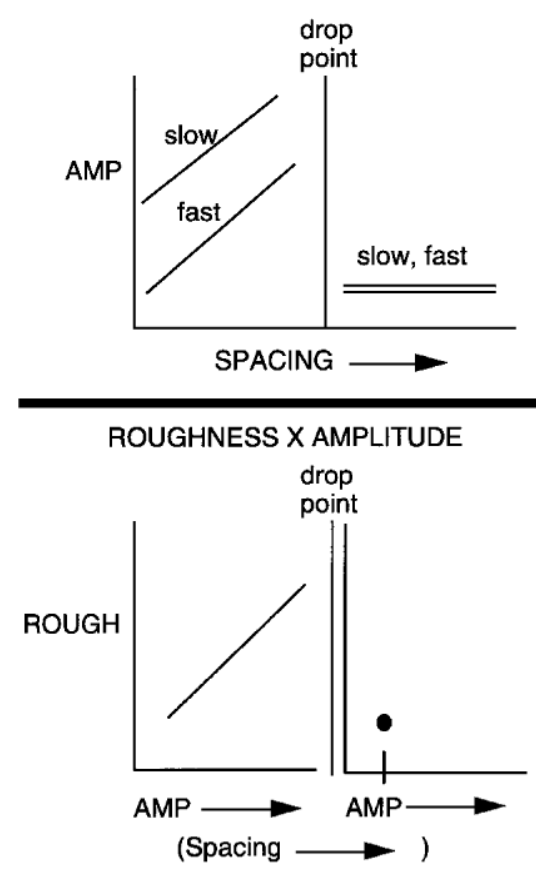

FREQUENCY $X$ SPACING \& SPEED

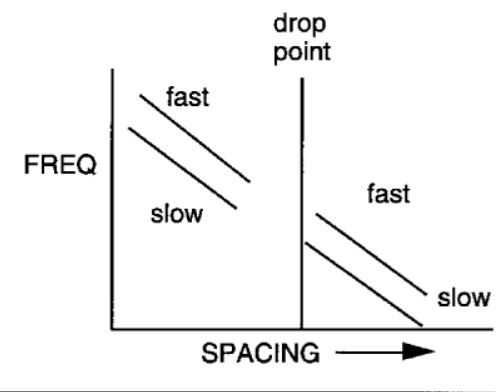

ROUGHNESS X FREQUENCY

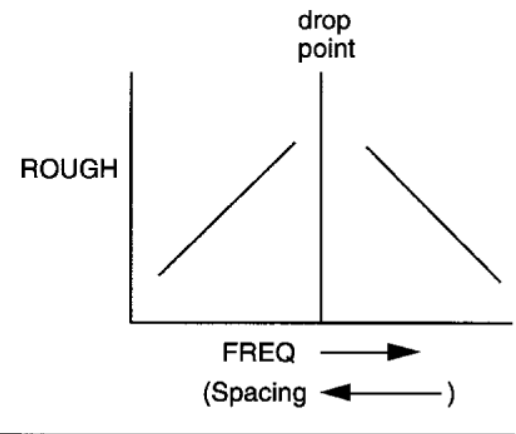

(Spacing $\longleftarrow$ )

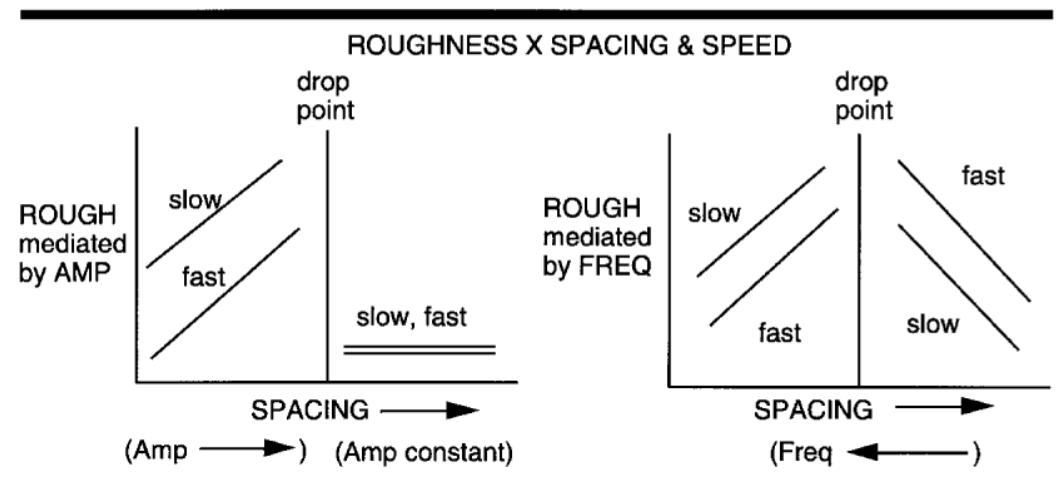

Figure 2. Analysis of effects of interelement spacing and exploratory speed on perceived roughness. Top panel: Effects of spacing and speed on amplitude (left panel) and frequency (right panel). Middle panel: Effects of amplitude and frequency on perceived roughness. The middle panel shows separate amplitude axes before the drop point in spacing and after it, where amplitude is depicted as having a constant value. Bottom panel: Resultant effects of spacing and speed on perceived roughness.

Taken together, the assumptions depicted in the top and middle panels of Figure 2 provide a qualitative account of our previous spacing and speed effects (Klatzky \& Lederman, 1999; Lederman et al., 1999), as depicted in the bottom panel. (1) When spacing effects below the drop point are considered, perceived roughness should increase with interelement spacing. By the foregoing assumptions, this trend reflects two underlying effects: Vibratory amplitude increases with increases in spacing, and frequency decreases. If roughness is directly related to amplitude and inversely related to frequency below the drop point, both effects will cause roughness to increase with spacing. (2) When speed effects below the drop point are considered, roughness should decrease with increases in speed. Again, there are two potential underlying effects. First, vibratory amplitude decreases as speed increases, and roughness varies directly with amplitude. Second, vibratory frequency will be higher at the fast speed, and given the inverse roughness/frequency relationship in this spacing range, roughness will be lower. (3) When spacing and speed effects above the drop point are considered, the analysis suggests that the previous effects will reverse. Amplitude will be relatively constant, and frequency will decrease with increases in spacing and increase with increases in speed. Since roughness is assumed to vary directly with fre- 

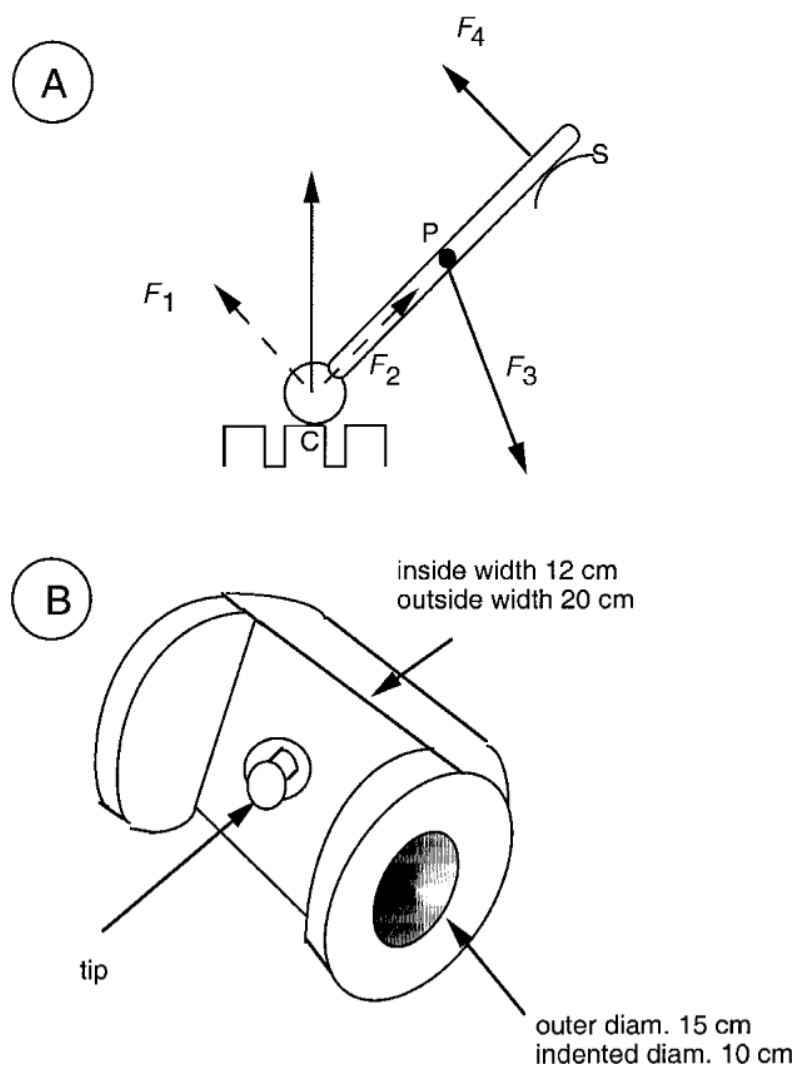

Figure 3. (A) Forces on a probe held between two fingers at Point $P$ and resting on the skin between them at Point $S$. See the text for explanation. (B) Zero-moment probe. The index finger and the thumb hold the recessed ends, which lie on a direct line with the probe tip.

quency in this range, perceived roughness should likewise decrease with increases in spacing and increase with increases in speed.

\section{Analysis of the Force Moment From a Stylus-Shaped Probe}

The third analysis of physical variables presented here concerns the posture with which a stylus-shaped probe is held and the resulting force moment. Suppose, as is shown in Figure 3A, that the probe is grasped at a point along its shaft $(\mathrm{P})$ by the thumb and the index finger, while the shaft rests on the skin between them at point $S$. The shaft forms an angle with respect to the surface. The fingers at the grasp point exert a force $\left(F_{3}\right)$ on the probe, causing the tip to exert a force on the surface. The surface exerts an equal and opposite force on the tip, which can be decomposed into components along the shaft $\left(F_{2}\right)$ and perpendicular to it $\left(F_{1}\right)$. The latter will generate a moment or torque around the grasp point, $\mathrm{P}$. The torque depends on the magnitude of the force $F_{1}$ and the distance of the grasp point from the probe tip. The torque is felt by the contacting parts of the hand as a system. It is the opposing directions of the forces around the grasp point that constitute the signal for torque. At point $P$, the effects of the exerted force will be sensed, and at point $\mathrm{S}$, the force generated by surface contact $\left(F_{1}\right)$ will be resisted by the skin contacting the shaft and felt as an opposing force $\left(F_{4}\right)$. The preceding describes contact with multiple points on the hand. However, because the skin holding the probe conforms to its surface, even a single finger comprises multiple contact points, and the user will sense opposing forces around the center of the grasp.

Note that in Figure 3A, if the index finger and the thumb were to move down the probe to the contact point (C), the moment would be eliminated, because the distance from the grasp point to the probe tip is zero (along the probe axis). Figure $3 \mathrm{~B}$ shows a zero-moment probe designed to eliminate the moment and restrict the locus of vibratory stimulation to the finger pads of the thumb and the index finger. This was used in two of the experiments reported here

\section{DATA ANALYSIS}

The present experiments provide data that examine the effects of exploratory and geometric variables. In Experiments 1 and 2, we used the zero-moment probe designed to eliminate force moments. In Experiment 1, this probe design was compared with the bare finger, and in Experiment 2 it was compared with the stylus-shaped probe used previously. Probe tip diameter was also manipulated in both experiments. In Experiments 3 and 4, we investigated the effects of probe diameter and exploratory speed, using the stylus probe with passive speed control. The two sets of experiments also differed in the geometry of the textured elements.

Because of the quadratic relation that we have found between perceived roughness and interelement spacing, given exploration with a probe, the data analysis for all the experiments focused on the parameters of a quadratic function fit to the magnitude estimations on $\log / \log$ scales. Although we assume that the underlying process for magnitude estimation produces a quadratic function, we do not mean to imply that a unitary process is involved. Consider that where $R$ denotes roughness and $s$ denotes interelement spacing, a quadratic of the form

$$
\log R=a *(\log s)^{2}+b *(\log s)+c
$$

will correspond to a function of the form

$$
R=10^{c} * s^{(a * \log s+b)} .
$$

As a process description, this would indicate that roughness is a power function of spacing, where the power itself contains log spacing as a component. Rather than assuming such a single underlying process, we assume that the quadratic reflects a qualitative change in processing at a critical spacing value, the drop point. Its parameters describe that underlying process and its change, as follows.

The parameters of the quadratic are (1) curvature (essentially, the rate at which the function approaches the peak), (2) peak height (the maximum ordinate of the fitted function), and (3) peak location (the abscissa value 
corresponding to the peak height). Where the quadratic is of the form $a x^{2}+b x+c$, the curvature is equal to $a$, the peak height is equal to $c-b^{2} / 4 a$, and the location of the peak on the $x$-axis is at $-b / 2 a$. Peak location is measured in millimeters of spacing, and peak height is measured in arbitrary, normalized-magnitude units. The curvature is taken as an index of sensitivity to the spacing continuum, because the greater the curvature, the greater the local slope of the function and, therefore, the greater the difference in magnitude corresponding to a given difference in spacing. (In a linear function without a quadratic term, the slope would be considered a measure of sensitivity by similar reasoning.) The peak height indicates the maximum roughness magnitude, and the peak location indicates the point at which the transition occurs from a positive relation between roughness and spacing to a negative one. Note that this parameterization does not depend on the range of the abscissa that is examined, unlike other parameters, such as the mean roughness or the slope over a restricted set of abscissa values.

The experimental technique used in these experiments, magnitude estimation, requires that subjects' data be individually normalized to remove effects of the individual's choice of scale. The nature of the normalization, however, can potentially affect what is retained about parameters of the underlying process. The procedure used here was intended to produce a function that preserves the meaning of these parameters but accommodates differences in the scales used by different subjects. It is based on three assumptions, as follows. First, the underlying perceptual process that generates a magnitude estimate follows a quadratic relation between perceived magnitude and interelement spacing on $\log / \log$ scales. Second, this quadratic relation is common to all subjects within a condition (as defined, e.g., by a probe diameter and element height), but the use of different scales by different subjects reflects different scale factors (i.e., multiplicative constants) that are applied to the output of the perceptual process. Third, the scaling differences among subjects are assumed to remain constant across different conditions. It can be shown that under these assumptions, the present data analysis preserves the quadratic and linear term of the underlying perceptual function in absolute terms, and the constant is preserved relatively across conditions. ${ }^{1}$ As a result, the data analysis preserves the absolute curvature and the location of the peak of the quadratic, as well as the relative height (difference in height between pairs of conditions within a single experiment). Violation of the above assumptions means that the data normalization does not preserve the parameters of the quadratic in the same way. In particular, if the scaling relation among subjects varies with condition, the quadratic and linear terms can be preserved, but not the constant. As a result, curvature and peak location can be preserved, but not the height of the function, even in relative terms. In short, if the process underlying vibratory roughness perception is intrinsically quadratic, under reasonable assumptions we will be able to capture the parameters of that process and compare them across conditions.

\section{EXPERIMENT 1 Zero-Moment Probe Versus Finger}

The quadratic relationship between perceived roughness and interelement spacing, when people explore surfaces with a probe, has been attributed to a transition in vibration at the spacing level at which the probe tip penetrates between elements (the drop point). Because the previous experiments of Klatzky and Lederman (1999) used stylus-shaped probes that were held like a pencil, vibration was accompanied by a force moment signaled to the hand. That is, the stylus made contact with the sides of the fingers and the pad of the thumb, along with the skin between the metacarpophylangeal joints of the index finger and the thumb. As is shown in Figure 3A, the forces from contact between probe tip and textured surface would lead to a moment around the fingers that would be felt by the skin sites in contact. Experiments 1 and 2 of the present paper were intended to eliminate the force moment and exclusively signal vibrations to the pads of the thumb and the index finger. They used a zeromoment probe that was held with a pinch grasp, which aligned the centers of the grasping fingers with the tip of the probe. Experiment 1 varied probe tip diameter while comparing the zero-moment probe with the finger, to determine whether the vibratory cues to the finger pads alone would lead to a quadratic relation between log interelement spacing and log roughness magnitude. It also compared the magnitude estimation function from the probe with one resulting from exploration with the bare skin.

\section{Method}

Subjects. The subjects were 12 students from Carnegie Mellon University. There were 3 females and 9 males. All were righthanded by self-report.

Apparatus and Stimuli. The stimuli were nine plates measuring $15.2 \times 2.5 \mathrm{~cm}$, fabricated by a plastic printing process $(\mathrm{Pad}$ Print Co.) that produced cylindrical raised elements. Negatives for the plates were created using an algorithm described previously (e.g., Klatzky \& Lederman, 1999). The design of each plate began with a graphic display of circular elements (dots) arranged in a matrix with equal distance between elements along horizontal and vertical rows. The elements were then displaced randomly by an algorithm that moved each dot some distance along an angular vector. The angle was randomly drawn from $0^{\circ}$ to $350^{\circ}$ in steps of $10^{\circ}$, and the distance was a randomly drawn proportion of the edge-to-edge distance between elements, constrained such that no element could move past the former position of another. The horizontal and vertical distances between the edges of the elements (i.e., not center to center) in the unjittered basic version of the matrix are shown for each plate in Table 1. This will be called the HV spacing (for horizontal and vertical) between elements; it has been used in previous reports by the authors. A different way of calculating the interelement spacing in the plates averages the distances from each element to the eight elements that lie adjacent along the diagonal, as well as horizontally and vertically; this will be called the HVD spacing. These spacings are also shown for each plate in Table 1. A 
Table 1

Interelement Spacing Values for the Plates Used in the Experiments, Based on the Seminal, Nonjittered Matrix of Elements, When Measured Horizontally and Vertically (HV) Including the Diagonal (HVD) Also

\begin{tabular}{ccc}
\hline HV Spacing $(\mathrm{mm})$ & $\begin{array}{c}\text { HVD Spacing }(\mathrm{mm}) \\
\text { (Experiments 1 and 2) }\end{array}$ & $\begin{array}{c}\text { HVD Spacing }(\mathrm{mm}) \\
\text { (Experiments 3 and 4) }\end{array}$ \\
\hline 0.500 & 0.778 & 0.699 \\
0.875 & 1.230 & 1.151 \\
1.250 & 1.683 & 1.604 \\
1.625 & 2.135 & 2.057 \\
2.000 & 2.588 & 2.509 \\
2.375 & 3.041 & 2.962 \\
2.750 & 3.493 & 3.414 \\
3.125 & 3.946 & 3.867 \\
3.500 & 4.399 & NA \\
\hline
\end{tabular}

microscope was used to measure five elements per spacing value to the nearest $0.1 \mathrm{~mm}$; by this means, the element diameter averaged $0.86 \mathrm{~mm}$ (cf. the specification by the computer algorithm of $0.84 \mathrm{~mm}$ ), and by the same technique, the element heights averaged $0.20 \mathrm{~mm}$.

The probe apparatus was a cylindrical device made of Delrin plastic, shown in Figure 1B. The center of the cylinder was cut out, leaving a flat surface into which was mounted a Delrin probe. Each probe had a spherically shaped tip with a designated diameter, mounted on a shaft about $16 \mathrm{~mm}$ in length (varying somewhat with tip diameter) that extended into the body of the cylinder, where it was secured with a hex screw. The probe tip diameters used in this experiment were 2,5 , and $8 \mathrm{~mm}$. Indentations in the ends of the cylinder allowed the subjects to hold it with their fingers placed so that the centers of the fingertips were aligned along an axis coinciding with the outer surface of the probe tip, which contacted the plate.
For presentation, a given plate was mounted on a block $2.5 \mathrm{~cm}$ wide. The ends of the plate were stabilized in niches, one of which slid back and forth to allow easy mounting of the plate. The upper surface of the block, which contacted the plate, was mounted with a thin rubber strip to minimize sliding.

Procedure. The subject wore a blindfold and headphones, through which were played taped sounds of probe/plate contact, to mask any sounds associated with exploration during the trial. Each subject explored the set of plates under four conditions: bare finger, 2-mm probe, 5- $\mathrm{mm}$ probe, and $8-\mathrm{mm}$ probe. The order of the conditions was counterbalanced across subjects. Within each condition, each plate was tested three times, with plate order entirely random within a condition.

On each trial, the block holding the plate was aligned so that the long dimension lay along the subject's frontal plane. The subject's hand, holding the probe or with the index finger extended, was guided to the plate. The subject was instructed to explore each plate with light force by running the finger or the probe along it about an inch in each direction; neither speed nor force was externally controlled. The subjects were instructed in the probe conditions to avoid contacting the sides of the block with the sides of the cylinder. When the subject was ready, he or she reported a number (excluding zero) indicating how rough the surface felt, with higher numbers used to indicate greater roughness. The experiment began with a series of practice trials that illustrated the range of plate valuesfive practice trials with each of the four probe conditions.

\section{Results}

Initially, the three estimates given by a subject within each condition were averaged. Then, to control for differences in numerical scale, the magnitude estimations for each subject were normalized by dividing each one by the subject's overall mean (over stimulus plates and conditions). The normalized estimates were then rescaled,

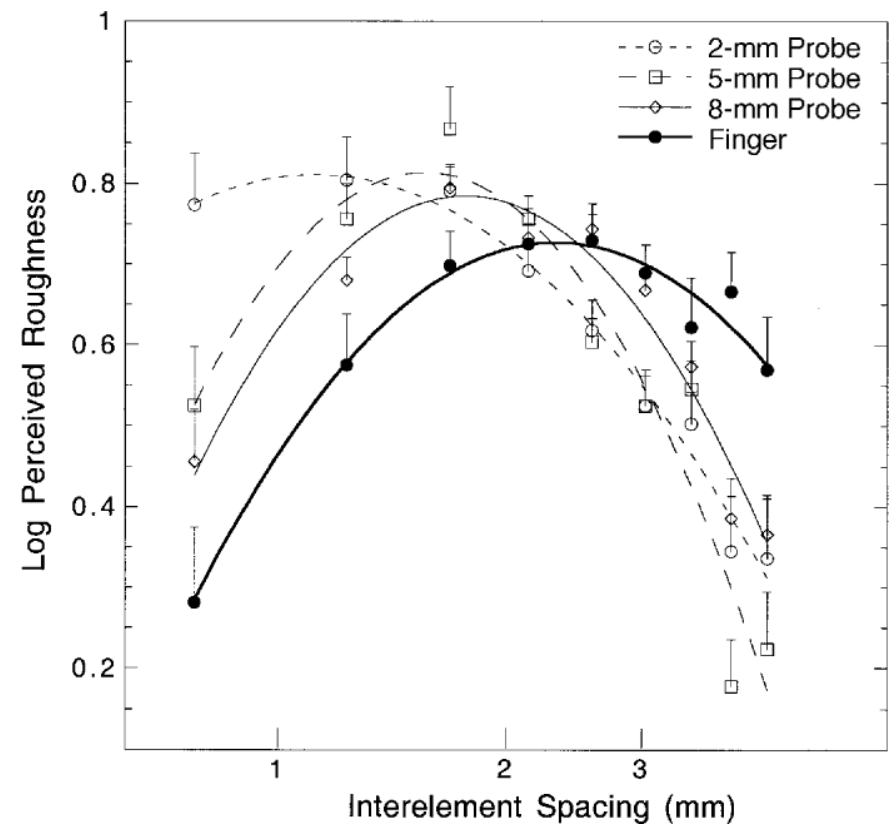

Figure 4. Log roughness magnitude by HVD spacing (in millimeters; $\log$ scale) in Experiment 1 for the finger and the three probe sizes. Quadratic functions have been fit to the data. Bars indicate $1 S E M$. 
to indicate the overall magnitude used, by multiplying by the grand mean across subjects, stimuli, and conditions. The data were then logarithmically transformed to produce more nearly normal distributions. As was noted above, this approach preserves key parameters of the processes that, in theory, underlie subjects' magnitude estimations. In Figure 4, the geometric means for the magnitude estimates are shown as a function of interelement HVD spacing (log scales) for each end effector.

As we found previously (Klatzky \& Lederman, 1999), the magnitude estimation functions for the probe were clearly quadratic in form; here, a quadratic trend was also observed for the finger across the spacing range. The $r^{2}$ values for the quadratic functions fit to the data in Figure 4 were all $\geq .95$. The curvature, peak height, and peak location parameters of the quadratic are shown in Table 2 (from a function fit to the mean data, and giving the antilog of peak location and height-i.e., the actual value of location in millimeters and the height in magnitude estimation units scaled by the mean).

One-way analyses of variance (ANOVAs) were performed across exploratory conditions (finger and 2-, 5-, and $8-\mathrm{mm}$ probe) on each of the parameters fit to the $\log / \log$ functions (curvature, peak location, and peak height), where the quadratic fit used the HVD spacings as the abscissa. (Except in the few cases noted, the results were the same when fit to the HV spacings.) Data from individual subjects yield unstable estimates of these parameters if the subject's function approaches a monotonic trend, in which case the quadratic coefficient (curvature) is near zero and can even be negative (i.e., the curve is concave upward). We eliminated a subject's observation if the curvature was negative, the estimate of peak location (which depends on both curvature and linear trend) in millimeters (i.e., antilog) was $\geq 25$, well beyond the range of the spacing values used (maximum $=$ 3.5 ), or the height was $>50$ on the antilog scale (well beyond the typical range of normalized magnitudes). Often, these criteria were redundant in indicating that an observation was unreliable. On this basis, in 3 out of 48 cases, we eliminated a subject's observation and substituted the mean over the remaining subjects.

The ANOVA for the curvature parameter was significant $[F(3,33)=10.91, p<.001]$, as was that for peak location $[F(3,33)=10.10, p<.001]$, but not for peak height $(p=.15)$. Post hoc least significant difference (LSD) tests indicated that the curvature values clustered into two sets: Finger and 2-mm probe (statistically equivalent) were less than 5- and 8-mm probes (which were equivalent). As for peak location, as was found by Klatzky and Lederman (1999), the peak of the function shifted rightward as the probe tip increased in diameter, with the finger yielding the highest estimate of all. Post hoc LSD tests showed that the peak location for the

Table 2

Parameters of the Quadratic Functions (Antilog Peak Location in Millimeters, Curvature, and Antilog Height) Fit to Mean Data for HVD and HV Spacing in Experiments 1 and 2, by Type of Effector, for Each Experiment and Group and Averaged Over Experiment and Group

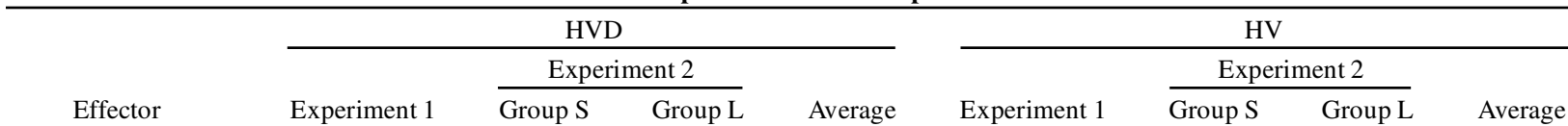

\section{Finger}

Zero moment, $2 \mathrm{~mm}$

Zero moment, $5 \mathrm{~mm}$

Zero moment, $8 \mathrm{~mm}$

Stylus, $2 \mathrm{~mm}$

Stylus, $5 \mathrm{~mm}$

Stylus, $8 \mathrm{~mm}$

\section{Finger}

Zero moment, $2 \mathrm{~mm}$

Zero moment, $5 \mathrm{~mm}$

Zero moment, $8 \mathrm{~mm}$

Stylus, $2 \mathrm{~mm}$

Stylus, $5 \mathrm{~mm}$

Stylus, $8 \mathrm{~mm}$

Finger

Zero moment, $2 \mathrm{~mm}$

Zero moment, $5 \mathrm{~mm}$

Zero moment, $8 \mathrm{~mm}$

Stylus, $2 \mathrm{~mm}$

Stylus, $5 \mathrm{~mm}$

Stylus, $8 \mathrm{~mm}$
5.31

6.48

6.72

6.09
2.32

1.12

1.69

1.76

$-1.95$

$-1.43$

$-2.98$

$-2.74$

8.09
5.91

1.04

1.61

0.54

1.44

$-1.56$

$-3.39$

$-0.81$

$-2.29$

7.09

6.79

Peak Location I

$-2.85$

$-1.43$

$-2.73$

Peak Height

$\begin{array}{ll} & 5.31 \\ & 6.79 \\ 6.45 & 6.65 \\ 5.68 & 5.89 \\ & 8.09 \\ 6.31 & 6.11 \\ 6.64 & 6.64\end{array}$

0.54

1.32

1.75

Curvature

$$
-1.95
$$$$
-1.50
$$$$
-2.82
$$$$
-2.80
$$$$
-0.81
$$$$
-1.86
$$$$
-2.73
$$

\section{0}

6.11

6.64
1.73

0.79

1.21

1.27

.32
.08

7

.

(2)

-1.54
-1.22
-2.43

$-2.19$

$-1.36$

5.28

6.59

6.85

6.14
$-2.76$

$-0.77$

$-1.93$

0.74

1.15

0.42

1.03

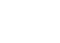

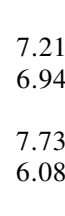

1.73

0.76

1.14

1.28

0.42

$\begin{array}{ll}0.84 & 0.94 \\ 1.26 & 1.26\end{array}$

$\begin{array}{ll}0.84 & 0.94 \\ 1.26 & 1.26\end{array}$

$-1.54$

$-1.29$

$-2.30$

$-2.25$

$-0.77$

$\begin{array}{ll}-1.23 & -1.58 \\ -2.21 & -2.21\end{array}$

\begin{tabular}{ll} 
& \\
& 5.28 \\
& 6.90 \\
6.56 & 6.78 \\
5.74 & 5.94 \\
& 7.73 \\
6.43 & 6.26 \\
6.74 & 6.74 \\
\hline
\end{tabular}


2-mm probe was lower than the others, the peak location for the finger was higher than the others, and the 5- and 8 -mm probes were statistically equivalent.

\section{Discussion}

Experiment 1 demonstrated that without a force moment, vibrations transferred from a rigid probe to the pads of the index finger and the thumb during exploration of a textured surface are sufficient to produce a percept of roughness that bears a regular relation to the geometry of the plate. Specifically, the log magnitude of perceived roughness is related to the log of interelement spacing in the stimulus by a quadratic function. The function can be described in terms of three parameters: curvature, which indicates overall sensitivity to the abscissa variable (here, interelement spacing), peak location, which indicates the abscissa value that gives the maximum roughness, and peak height, the maximum roughness achieved.

Not only the probe-produced functions, but also the function for the finger showed a clear downturn in roughness magnitude over the range of elements tested. The function for the finger is usually linear in this range, although it can reverse at larger spacings. For example, Connor and Johnson (1992) found a downturn when HV spacings were greater than $3.5 \mathrm{~mm}$, which is above the spacing value at which the presently obtained peaks occurred. On the other hand, using densely spaced columns of raised dots $1.8 \mathrm{~mm}$ high, Smith et al. (2002) found monotonic trends over variations in spatial period up to $10 \mathrm{~mm}$. A factor that is likely to contribute to the peak at an early spacing may be the height of the present elements, which was substantially lower than that used in our 1999 studies (Klatzky \& Lederman, 1999; Lederman et al., 1999) or in that of Smith et al. The foregoing analysis of probe/plate geometry (Equation 1) shows how the height of the raised elements on a plate affects the drop point. According to the analysis, the lower height of the present elements, relative to those used in 1999, should cause the drop point to occur earlier on the continuum, which according to our analysis should similarly affect the peak of the magnitude estimation function. Although this analysis was formulated for exploration with the probe, the skin of the finger should similarly "bottom out" earlier with the shallower heights.

As we found in our previous experiments with stylus probes (Klatzky \& Lederman, 1999; Lederman et al., 1999), the peak of the quadratic function moved rightward along the spacing dimension as the probe tip became larger. The function obtained from exploration with the finger had the rightmost peak, in keeping with its having the largest surface size among the effectors used here. The correspondence of the peak location and the geometrically determined drop point will be considered further in the General Discussion section.

The curvature value was reliably greater for the large probes ( 5 and $8 \mathrm{~mm}$ ) than for the finger and the $2-\mathrm{mm}$ probe. Thus, larger probe sizes produced relatively greater sensitivity to the spacing in the stimuli, but the relation between effector size and sensitivity did not extend to the finger, which had the largest contact surface but not the greatest sensitivity. Although the effect of probe size on peak height was not significant, the 8-mm probe produced the lowest height, which is consistent with findings from the other experiments, reported next.

\section{EXPERIMENT 2 Zero-Moment Probes Versus Stylus}

Experiment 2 was conducted to compare perceived roughness with the zero-moment probe with that with the stylus-shaped probe used by Klatzky and Lederman (1999). The distal geometry of the probe/plate interaction is held constant across the two types of probe, but the probe/finger interaction differs. The zero-moment probe eliminates force moments. Moreover, it transmits vibrations from the probe-tip/plate interaction to the finger pads, whereas the stylus, which is held like a pencil, sends the vibratory signal from the plate to the sides of the fingers and the skin between the index finger and the thumb. In addition, the differences in how the probes are gripped might lead to differences in the effects of exploratory factors, such as speed and force, which affect the frequency and amplitude of vibration.

We wished to determine whether these various factors would change the perception of roughness and, if so, how. The sensitivity to spacing variations in the stimuli might be greater for the zero-moment probe, due to the contact with the finger pads. This would increase the curvature of the quadratic magnitude estimation function. The overall roughness magnitude and the peak magnitude might be greater for the stylus probe, due to the additional points of skin contact and the presence of the force moment. The location of the peak along the spacing axis would not be affected if probe/plate geometry were the controlling factor; however, peak location could be affected if the grip differences led the subjects to move the probes at different speeds. The direction of this effect could not be predicted a priori.

\section{Method}

Subjects. The subjects were 24 students from the same population as that in Experiment 1. To reduce experimental demands, they were divided into two groups, varying in the region within the size continuum of the probe tips used. There were 4 females and 8 males in the group with probes from the smaller size region and 3 females and 9 males in the group with probes from the larger region. An additional 2 subjects in each group were eliminated because they showed a monotonic trend relating roughness magnitude to interelement spacing in every condition and debriefing indicated they were trying to count the elements, rather than report roughness.

Procedure and Design. The plates were the same as those used in Experiment 1. There were two types of probes: zero moment and stylus. The total length of the stylus was $13 \mathrm{~cm}$, the last $28 \mathrm{~mm}$ of which consisted of a thinner, tapering shaft culminating in a spherical tip with a designated diameter. The remainder of the stylus, which was held by the subject, was a cylindrical shaft $9 \mathrm{~mm}$ in diameter. The subject held the zero-moment probe as in Experiment 1 ; the stylus probe was held like a pencil. 

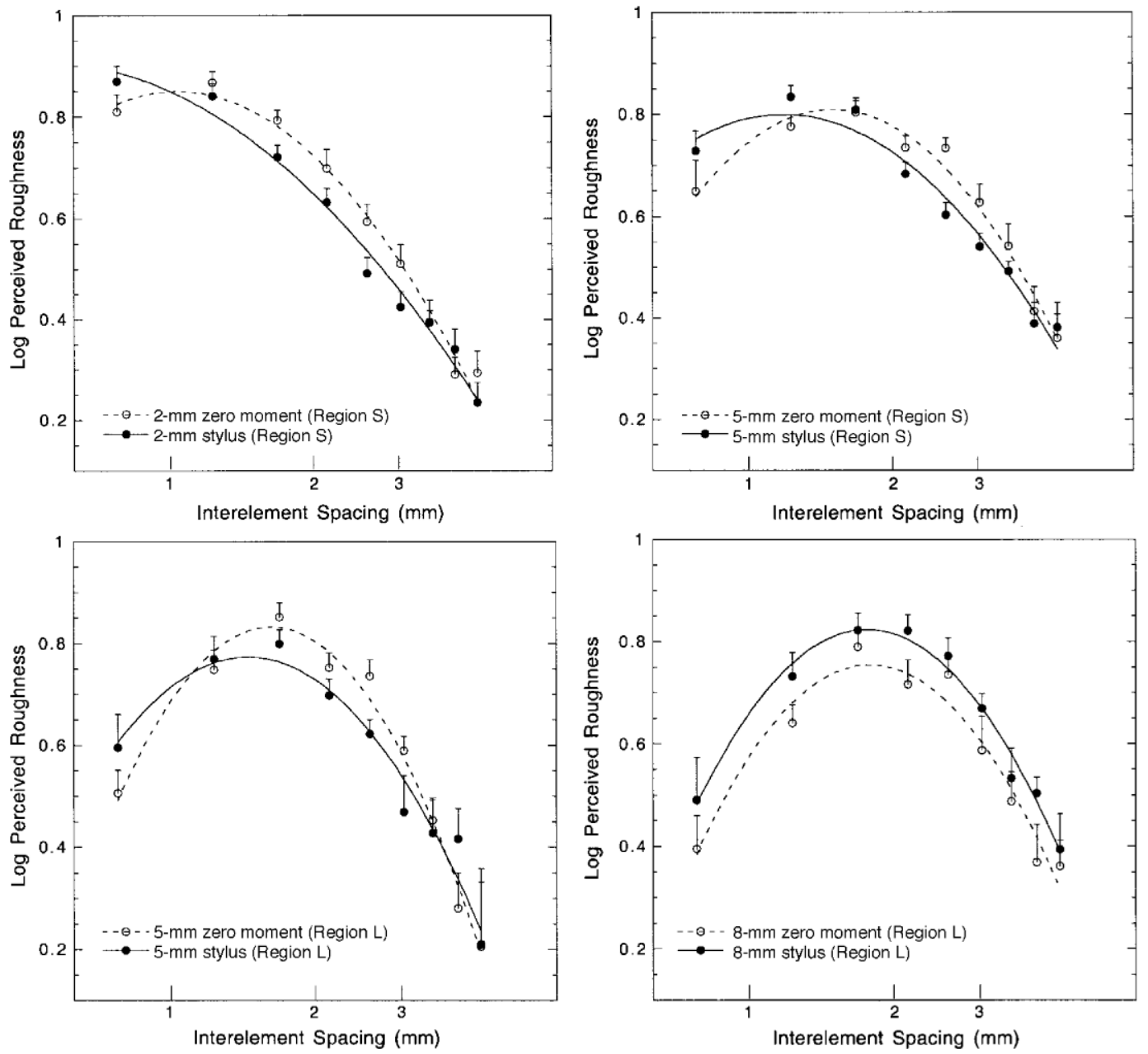

Figure 5. Log roughness magnitude by HVD spacing (in millimeters; log scale) in Experiment 2. Quadratic functions have been fit to the data. Bars indicate $1 S E M$. Each panel represents a zero-moment and a stylus probe at one size. Region S: 2- and 5-mm sizes; Region L: 5- and 8-mm sizes.

The subjects took part in four conditions, constituting a crossing of two probe tip diameters and two probe types (zero moment vs. stylus). An additional between-subjects variable was the region in which the tip diameters fell: The subjects with the smaller size region (S) had tip diameters of 2 and $5 \mathrm{~mm}$; those with the larger size region (L) had tip diameters of 5 and $8 \mathrm{~mm}$. Thus, the 5 - $\mathrm{mm}$ tip was common to both groups. This division into two groups was used to reduce the number of judgments made by any 1 subject.

The design was like that of Experiment 1, with the four conditions tested in counterbalanced order across subjects and three replications of each plate within a condition, randomly ordered within conditions. As before, there were practice trials illustrating each condition at the beginning of the experiment. The instructions for exploring the stimuli were the same as those in Experiment 1.

\section{Results}

The relation of log magnitude to HVD spacing (log scale) is shown in Figure 5. The $r^{2}$ values for the quadratic functions in the figure were all $\geq .95$. To determine whether a grand mean across groups could be used to scale all the subjects' responses, the two groups of subjects, who differed in size region, were compared on the 5-mm probe conditions, which were common to both, using the $\log$ of the nonnormalized magnitudes. Although Figure 5 suggests some differences in the shape of the functions, the ANOVA on size region, plate, and probe type revealed no region effect (only an effect of plate and a plate $\times$ probe type interaction) in the directly comparable conditions $(p>.25)$, so the data were normalized as in Experiment 1, using the grand mean across groups.

ANOVAs were performed on the parameters of the quadratic equations fit to the functions relating $\log$ roughness magnitude to log HVD spacing, shown in Table 2. The factors were size region (S vs. L), probe type (zero moment vs. stylus), and probe size (large vs. small). Note that for region $\mathrm{S}$, the small probe was $2 \mathrm{~mm}$ in diameter, and the large was $5 \mathrm{~mm}$; with region $\mathrm{L}$, the small probe was $5 \mathrm{~mm}$ and the large was $8 \mathrm{~mm}$. One subject failed the tests described above for quadratic trend in 
one condition, so the mean over other subjects was substituted. Given the nature of our design, an interaction between group (relatively large vs. small probes) and another factor indicates that the effects of the latter factor differ across the region of probe sizes. Such interactions were probed with further tests.

With respect to the ANOVA on curvature, the measure of sensitivity to interelement spacing, the results indicated that larger probes produced greater curvature and that there was greater curvature for the zero-moment probe than for the stylus. Thus, there were main effects of size region $[F(1,22)=7.53, p<.05]$ and probe type $[F(1,22)=18.24, p<.001]$. Probe size and type also interacted $[F(1,22)=5.50, p<.05]$, and region further interacted with size and type $[F(1,22)=5.07, p<.05(p=$ .053 with HV spacing)]. These interactions reflect the finding that the $2-\mathrm{mm}$ probes produced the curvature with smallest magnitude and that the curvature for the zero-moment probe was greater than that for the stylus, especially for the 2- and 5-mm sizes. Tests comparing the small and the large probes within each region showed that the difference between the 2- and the 5-mm sizes with respect to quadratic curvature was significant for both the zero-moment probe and the stylus $[t \mathrm{~s}(11)=2.67$ and 2.66, respectively; $p$ s $<.025$ ]. However, the difference between the 5- and the 8 -mm sizes was not significant for either probe type.

With respect to peak location, all three main effects were significant [region, $F(1,22)=22.25, p<.001$; probe type, $F(1,22)=9.16, p<.01$; probe size, $F(1,22)=$ $28.45, p<.0001]$. The location of the peak moved rightward as probe size increased, producing the region and the size effects. The difference between peak location values with 2 - and 5-mm probes was greater than that with 5- and 8-mm probes, as was evident in Experiment 1 ; this was indicated by an interaction between region and probe size $[F(1,22)=11.32, p<.01]$. The size $\times$ type interaction was also significant $[F(1,22)=6.21$, $p<.05]$, reflecting the fact that the effect of probe diameter was greater for the stylus than for the zero-moment probe. Tests revealed that the $2-\mathrm{mm}$ probe produced a lower peak location than did the 5-mm probe for both the stylus and the zero-moment probe $[t \mathrm{~s}(11)=4.14$ and 4.43 , respectively; $p s<.01$ ]; however, the difference between peak locations for the 5 - and the 8 -mm probes was significant only for the stylus $[t(11)=2.28, p<.05]$. The type $\times$ region interaction was marginal $(p=.057$; this reached significance in the HV spacing analysis, $p<.05$ ). This interaction reflects the fact that the zero-moment probe tended to peak at a higher spacing value than did the stylus, but this difference decreased as probe size increased. To verify the trend directly, we tested the difference in peak location between the stylus and the zeromoment conditions at each probe size. The difference was significant for the $2-\mathrm{mm}$ probe [region $\mathrm{S} ; t(11)=$ $3.14, p<.01$ ] and the $5-\mathrm{mm}$ probe [both regions combined; $t(23)=2.23, p<.05$ ], but not for the $8-\mathrm{mm}$ probe (region L).
The analysis of the height of the quadratic, or peak roughness, showed a just-significant effect of size $[F(1,22)=4.36, p<.05$; this yielded $p=.087$ in the HV spacing analysis]. Height decreased as the probe increased in size for three of four comparisons, but only the 2-versus 5-mm comparisons for both probe types were significant by $t$ tests isolating the comparisons of interest [for the zero-moment probe, $t(11)=2.19$; for the stylus, $t(11)=2.13 ; p \mathrm{~s}<.05]$.

\section{Discussion}

Experiment 2 confirmed the basic findings of Experiment 1 , as follows. The zero-moment probe, like the stylus, produced a regular function relating log perceived roughness to log interelement spacing. The function was well fit by a quadratic, and the peak of the quadratic moved rightward (toward a greater spacing value) as the probe tip increased in size. The effect of probe size on peak location tended to saturate as the probe tip increased beyond $5 \mathrm{~mm}$, however. The distance between peak locations, in terms of interelement spacing, was greater when the 2 - and the 5-mm probes were compared than when the 5- and the 8-mm probes were compared.

Probe diameter affected other parameters of the quadratic as well, and as with peak position, the effects were greater in the smaller region of diameters. The curvature was smaller in magnitude for the $2-\mathrm{mm}$ probe than for the 5- and the $8-\mathrm{mm}$ probes, and the height decreased as the probe size increased from 2 to $5 \mathrm{~mm}$, but not beyond. Because the effects were greatest for small probes, in Experiments 3 and 4 we further examined the effect of tip diameter over a set of smaller values.

Experiment 2 also showed a difference in the functions fit to the zero-moment and the stylus probes, particularly when the probe size was small $(2$ and $5 \mathrm{~mm})$. The zeromoment probe produced greater curvature, indicating greater sensitivity to the spacing variable. The increased sensitivity may occur because the fingertips contact the zero-moment probe, whereas the sides of the finger contact the stylus probe, which is held like a pencil.

An additional difference between probes is that the quadratic functions fit to the data peaked later for the zero-moment probe than for the stylus. This finding suggests that the drop point for the zero-moment probe is at a wider spacing than that for the stylus. Because the probe tips are identical, this result indicates that factors other than probe tip geometry affect the drop point. It seems likely that differences in exploration with the two probe types affect the drop point. In theory and as we have observed previously (Lederman et al., 1999), the drop point should move to a wider spacing as exploratory speed increases, because there is less time for the probe tip to drop as it makes the transition between elements in the surface. If speed underlies the differences in peak location for the two probe types, the subjects must have explored more quickly with the zero-moment probe; further research would be needed to address this issue. In Experiment 4, speed effects were assessed in 
combination with variations in probe tip diameter, using the stylus probe.

Finally, although we anticipated that the force moment and additional skin contact might cause the overall roughness magnitude or the peak of the quadratic to be greater for the stylus probe, no effect of this type was obtained.

\section{EXPERIMENT 3 Size Effects With Stylus Probe}

In Experiments 3 and 4, an experimental apparatus allowed speed and force to be specified by the experimenter. A target force was executed under the subject's active control, and a target speed was controlled passivelythat is, directly by the apparatus. The stimuli were not those used in Experiments 1 and 2 but, rather, the plates used previously by Klatzky and Lederman (1999). In Experiment 3 , we addressed the effects of probe tip diameter variations, using the stylus probe, where diameter was varied within a smaller size region than in Experiments 1 and 2 . The goal was to verify that the peak of the quadratic would move rightward as the probe diameter increased and to compare the peak location with the geometrically determined drop point. We also wished to determine the effects of probe diameter variations within this smaller region on the height and curvature parameters. In Experiment 4, we addressed the effects of speed in combination with probe tip diameter.

\section{Method}

Subjects. The subjects consisted of 20 students, 15 women and 5 men, from Queen's University, who received course credit for their participation. All subjects were right-handed by self-report.
Apparatus. A force- and speed-control apparatus (Lederman, 1983; Lederman et al., 1999) was employed in the present experiment. It functioned like a traditional balance scale. At one end of the balance arm was a platform on which the stimulus plates rested. At the other end was a $0.29-\mathrm{N}$ weight. The subject placed his or her right arm in an armrest, with the wrist supported to prevent fatigue of the arm and to keep it stationary. The subject applied a counterforce of $0.29 \mathrm{~N}$ at the stimulus end to keep the balance arm steady and level as it moved under the stationary hand. The balance arm was linked to a motor, set on an adjacent table, so that it moved horizontally back and forth. The average speed of motion, controlled by a tachometer, was set to $115 \mathrm{~mm} / \mathrm{sec}$. The motion itself was sinusoidal, since the plate came to a stop and reversed direction at the end of each pass, which produced a peak speed of approximately $180 \mathrm{~mm} / \mathrm{sec}$.

The stimuli consisted of eight polymer plates with raised elements, produced using the Nyloprint photoengraving technique. The dots were shaped in the form of truncated cones, with a diameter of $0.46 \mathrm{~mm}$ at the top and an average base diameter of $1.05 \mathrm{~mm}$ (the base diameters varied somewhat with interelement spacing, due to the production process). The height of the cone was $0.40 \mathrm{~mm}$. These measurements were based on microscopic examination of five elements per spacing value. Dot spacing was computer generated with the same algorithm as that described in Experiment 1; the spacing in this case applied to edge-to-edge distances between the tops of the truncated cones. The eight interelement spacings ranged from 0.5 to $3.125 \mathrm{~mm}$, in $0.375-\mathrm{mm}$ increments. The corresponding HVD spacings are shown in Table 1. The probes were in the shape of a stylus with a shaft $11 \mathrm{~cm}$ long and $9 \mathrm{~mm}$ in diameter, terminating in spherical ends with diameters of 1.0, 2.0, and $3.0 \mathrm{~mm}$.

Procedure. Sound abatement procedures were similar to those in Experiments 1 and 2, using earplugs and a tape of probe/plate contact sounds. The subject rested his or her right arm on the armrest, so that the right hand was above the platform supporting the plate. The probe shaft was gripped like a pencil. Initial practice was given with 12 different combinations of probe sizes and interelement spacings, varying broadly across the stimulus range. On each trial,

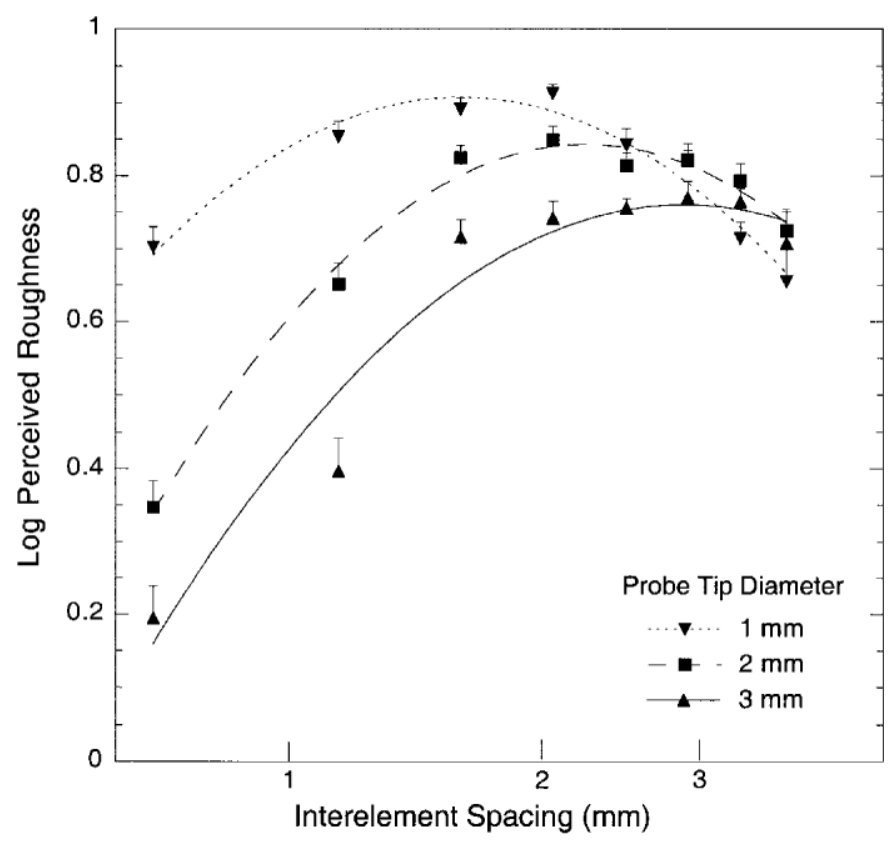

Figure 6. Log roughness magnitude by HVD spacing (in millimeters; $\log$ scale) in Experiment 3, for three probe sizes. Quadratic functions have been fit to the data. Bars indicate $1 S E M$. 
Table 3

Parameters of the Quadratic Functions (Antilog Peak Location in Millimeters, Curvature, and Antilog Height) Fit to Mean Data for HVD and HV Spacing in Experiments 3 and 4, by Probe Diameter and Speed and Averaged Over Speeds

\begin{tabular}{|c|c|c|c|c|c|c|c|c|}
\hline \multirow[b]{3}{*}{ Effector } & \multicolumn{4}{|c|}{ HVD } & \multicolumn{4}{|c|}{$\mathrm{HV}$} \\
\hline & \multirow{2}{*}{$\begin{array}{c}\text { Experiment } 3 \\
\text { (Medium } \\
\text { Speed) }\end{array}$} & \multicolumn{2}{|c|}{ Experiment 4} & \multirow{2}{*}{$\begin{array}{c}\text { Averaged } \\
\text { Over } \\
\text { Speed }\end{array}$} & \multirow{2}{*}{$\begin{array}{c}\text { Experiment } 3 \\
\text { (Medium } \\
\text { Speed) }\end{array}$} & \multicolumn{2}{|c|}{ Experiment 4} & \multirow{2}{*}{$\begin{array}{c}\text { Averaged } \\
\text { Over } \\
\text { Speed } \\
\end{array}$} \\
\hline & & $\begin{array}{c}\text { Fast } \\
\text { Speed } \\
\end{array}$ & $\begin{array}{c}\text { Slow } \\
\text { Speed } \\
\end{array}$ & & & $\begin{array}{c}\text { Fast } \\
\text { Speed } \\
\end{array}$ & $\begin{array}{c}\text { Slow } \\
\text { Speed } \\
\end{array}$ & \\
\hline \multicolumn{9}{|c|}{ Peak Location } \\
\hline $1 \mathrm{~mm}$ & 1.61 & 1.96 & 1.31 & 1.63 & 1.22 & 1.52 & 0.99 & 1.24 \\
\hline $2 \mathrm{~mm}$ & 2.25 & & & 2.25 & 1.77 & & & 1.77 \\
\hline $3 \mathrm{~mm}$ & 2.93 & 3.67 & 2.50 & 3.03 & 2.42 & 3.14 & 1.99 & 2.52 \\
\hline \multicolumn{9}{|c|}{ Curvature } \\
\hline $1 \mathrm{~mm}$ & -1.65 & -1.26 & -1.60 & -1.50 & -1.44 & -1.08 & -1.41 & -1.31 \\
\hline $2 \mathrm{~mm}$ & -1.95 & & & -1.95 & -1.66 & & & -1.66 \\
\hline $3 \mathrm{~mm}$ & -1.55 & -1.06 & -1.29 & -1.30 & -1.28 & -0.87 & -1.09 & -1.08 \\
\hline \multicolumn{9}{|c|}{ Peak Height } \\
\hline $1 \mathrm{~mm}$ & 8.08 & 10.40 & 9.14 & 9.21 & 8.08 & 10.37 & 9.20 & 9.22 \\
\hline $2 \mathrm{~mm}$ & 6.96 & & & 6.96 & 6.91 & & & 6.91 \\
\hline $3 \mathrm{~mm}$ & 5.76 & 8.44 & 7.09 & 7.10 & 5.73 & 8.48 & 7.05 & 7.09 \\
\hline
\end{tabular}

the experimenter placed the probe into the subject's hand and allowed the platform to rise until the probe tip, guided by the experimenter, was gently brought into contact with the left end of the surface; thus, in the first pass, the platform moved to the left.

The experimental trials included two replications of the 24 combinations of probe diameter (3) and interelement spacing (8). Within each replication, the 24 trials were presented in a random order, with the constraint that no probe diameter could be presented in more than 3 consecutive trials.

\section{Results and Discussion}

The data from the two replications were combined for normalization and log transformation, performed as in Experiments 1 and 2, and are presented as a function of spacing in Figure 6. The $r^{2}$ values for the quadratic functions were all $\geq .94$. As before, we performed ANOVAs with probe size as a factor on the three components of the quadratic: curvature, peak location, and peak height. Table 3 gives the mean values of these parameters, where the peak location and height are reported as antilogs of the fitted values. We report ANOVAs using the HVD values and note any differences from the effects found with the HV values. Curvature showed no effect of probe size, in contrast to Experiments 1 and 2. Peak location did vary with probe size $[F(2,38)=32.57, p<.0001]$. As before, the peak moved rightward for the larger probes; post hoc LSD tests showed that all the means differed significantly. Peak height also showed a significant effect $[F(2,38)=9.64, p<.001]$. The height of the function decreased as the probe size increased; post hoc LSD tests showed that all the means differed significantly.

This experiment confirmed, with smaller probes, passive presentation, and different-shaped plate elements, that $\log$ roughness is a quadratic function of log spacing when a surface is explored with a stylus and that the location of the peak shifts rightward as the stylus tip size increases. The previously observed trend toward decreasing height of the functions with increasing probe size was also confirmed. However, this experiment did not replicate the previous finding that the smallest probe produced the smallest curvature, which was found for stylus-shaped and zero-moment probes in Experiments 1 and 2 . This point will be discussed below. The shapes of the functions mean that probe tip diameter had a large effect at dense spacings but that the probes were equivalent at the largest spacing. This is consistent with our analysis of drop point geometry (introduction and Figure 1), since all probes should be riding on the substrate with the largest spacing value.

\section{EXPERIMENT 4 Speed and Size Effects With Stylus Probe}

As was summarized above, the effects of speed on texture perception with a probe were previously examined by Lederman et al. (1999). In their experiments, a single probe tip diameter $(3 \mathrm{~mm})$ and two ranges of average speed (20-207 mm/sec and 55-222 mm/sec) were used. In addition, they compared active with passive control of speed. The passive control was as described for Experiment 3 and used here; with the active control, the subject was taught to move the stylus rhythmically to auditory cues. If, with active control, subjects use knowledge of efferent commands to compensate for the effects of speed on vibratory frequency, speed effects should be reduced. Passive control undoubtedly gives subjects some information about speed as well, through the duration of a pass across the plate and the peak vibration that is felt. Thus, passive cues might also be used to achieve speed constancy.

The results of Lederman et al.'s (1999) experiments showed that log roughness magnitude was a quadratic function of log spacing for both speed ranges and for active and passive control. An effect of speed was evident for both active and passive conditions, although it was slightly more pronounced for the passive. The magnitude of the speed effect was comparable to that reported by 
Lederman (1983) for exploration with the bare finger. The parameters of the quadratic were affected by speed as follows. The location of the peak roughness magnitude moved rightward along the spacing axis as the speed increased. Although Lederman et al. (1999) did not report an analysis of the curvature and height parameters, subsequent analyses showed that the magnitude of the curvature parameter tended to decrease with increasing speed (significantly so only with the larger speed range) and that the peak roughness (maximum height of the function) increased with increasing speed in one of the two experiments reported (the smaller speed range). A salient result of these parametric variations is that speed interacted with spacing in a crossover pattern, as was described previously.

In the present experiment, the extreme values of the smaller speed range used by Lederman et al. (1999) were used in combination with two probe diameters. We wished to replicate the effects of speed reported previously and to determine whether speed effects varied with probe tip diameter within this smaller range. Both speed and probe size should affect the drop point, which should shift the location of the peak of the magnitude estimation function.

\section{Method}

The subjects consisted of 20 students, 14 women and 6 men, from the same pool as that in the previous experiments. They were right-handed by self-report.

The same apparatus was used in this experiment as in Experiment 3. The experiment involved four withinsubjects factors: probe diameter, interelement spacing, speed, and repetitions. There were two probe diameters, 1.0 and $3.0 \mathrm{~mm}$, and the same eight plates were used as in Experiment 3. There were two speeds of presentation, $222 \mathrm{~mm} / \mathrm{sec}$ (fast) and $55 \mathrm{~mm} / \mathrm{sec}$ (slow); these correspond to peak speeds of 347 and $86 \mathrm{~mm} / \mathrm{sec}$, assuming sinusoidal movement. These speeds were the extreme values used by Lederman et al. (1999, Experiment 2); the speed of $115 \mathrm{~mm} / \mathrm{sec}$ used in Experiment 3 lay between these values and was also used in Lederman et al.'s (1999) study. All 32 combinations of probe diameter (2), speed (2), and interelement spacing (8) were carried out within each of two blocked replications. Within each block, the trials were in random order, with the same constraint as that in Experiment 3.

\section{Results}

The data were combined across replications, then normalized and log transformed as described previously. Analyses were performed on the parameters of the quadratics fit to the roughness/spacing functions, with factors of speed and probe size. The data and functions are shown in Figure 7, and the parameters are shown in Table 3. The $r^{2}$ values for the quadratic functions in Figure 7 were all $\geq .97$. Again, we report results for HVD spacing; HV effects were comparable, unless noted otherwise. Data for three cells were eliminated by criteria given above; the means for other subjects were substituted. The ANOVA for curvature showed a significant effect of speed $[F(1,19)=5.08, p<.05$; this did not reach significance in the analysis of HV spacing], such that curvature was greater for the slower speed. The ANOVA for peak location showed effects of size $[F(1,19)=$ $83.93, p<.0001]$ and speed $[F(1,19)=26.55, p<.001]$. As has been found consistently, the peak location moved rightward (sparser spacing) as the probe tip increased in size, and as predicted, it moved rightward as the speed

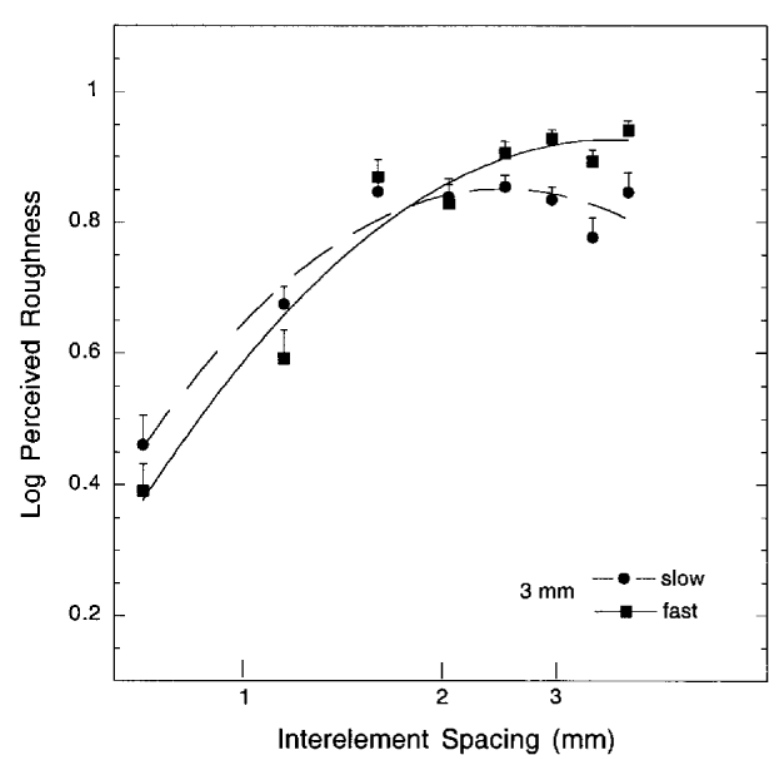

Figure 7. Log roughness magnitude by HVD spacing (in millimeters; log scale) in Experiment 4, for two probe sizes and two speeds. Quadratic functions have been fit to the data. Bars indicate 1 SEM. 
increased. The ANOVA for peak height also showed effects of size $[F(1,19)=97.28, p<.0001]$ and speed $[F(1,19)=12.00, p<.01]$. Peak height was greater for smaller tip size and faster speed.

\section{Discussion}

As was found in Experiment 3, log roughness was a quadratic function of log spacing. The location of the peak shifted rightward and the peak height decreased as the stylus tip diameter increased. Like Experiment 3 and in contrast to Experiments 1 and 2, tip diameter did not affect curvature.

The new contributions of Experiment 4 pertain to the effects of exploratory speed on the parameters of the quadratic function. The speed effects found here are similar to those in Lederman et al. (1999), in that roughness decreased with increases in speed at dense spacing values but increased with increases in speed for sparse ones.
The point of reversal of speed effects was near the peak of the magnitude estimation function, as is consistent with the idea that both reflect the transition in the vibratory input at the drop point (see Figure 2). Moreover, as was found by Lederman et al. (1999), the peak location moved rightward as the speed increased. As was further suggested by that study, curvature was greater in magnitude for the slow speed, and peak height was greater for the faster speed. Speed did not interact with probe tip diameter in any of the analyses of the quadratic parameters.

\section{GENERAL DISCUSSION}

The discussion will first summarize the effects on the quadratic magnitude estimation function that are common to these and related studies. Then the relation between the location of the peak of the magnitude estimation function along the spacing axis and the drop point will be consid-

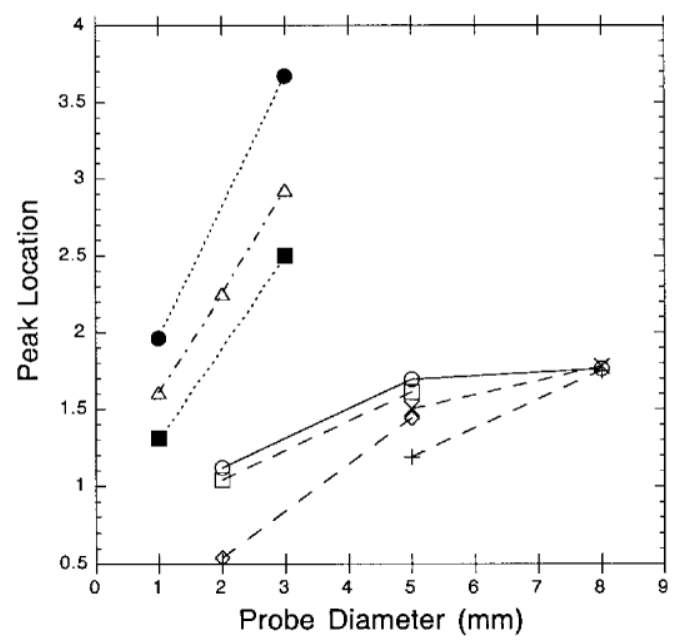

$$
\begin{aligned}
& -\odot 1 \text { zero moment } \\
& -\square \cdot 2-S \text { zero moment } \\
& -\diamond \cdot 2-S \text { stylus } \\
& -\star \cdot 2-L \text { zero moment } \\
& -+\cdot 2-L \text { stylus } \\
& -A \cdot 3 \text { stylus } \\
& \cdots \cdots \text { 4-fast stylus } \\
& \cdots \cdots \text { 4-slow stylus }
\end{aligned}
$$
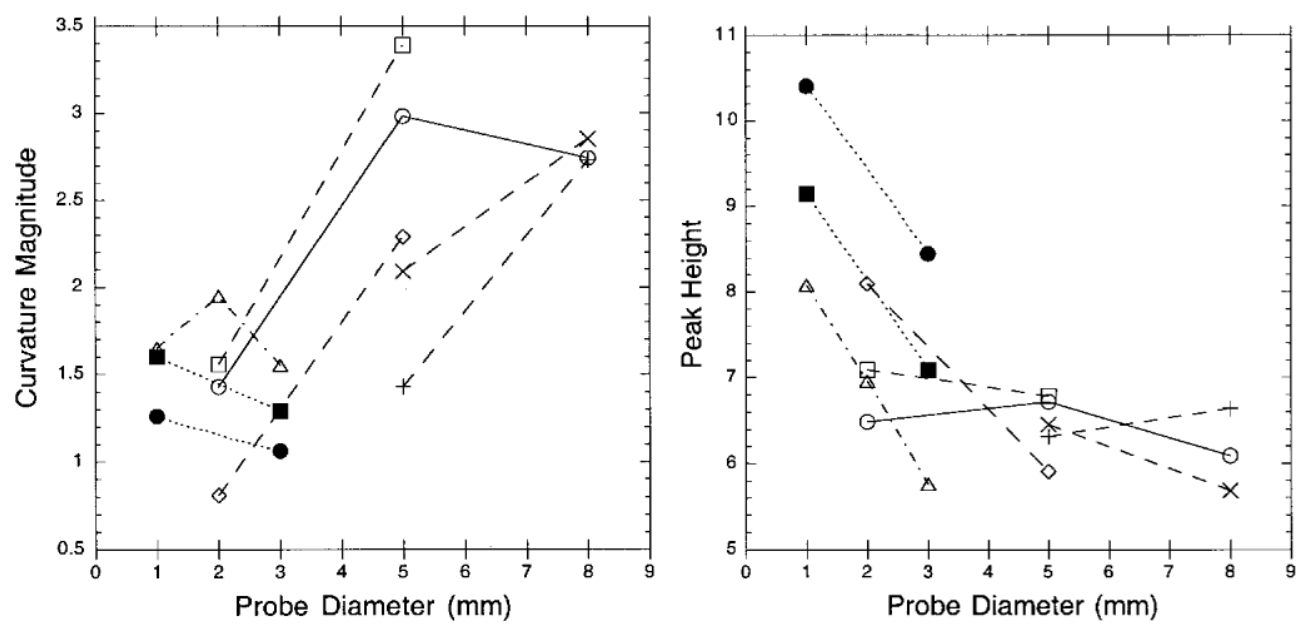

Figure 8. Values of peak location (in millimeters), curvature magnitude, and peak height, as a function of probe diameter (in millimeters) for Experiments 1-4. Lines connect conditions that differ only in diameter. Lines with a common graphic symbol come from the same experiment. Numbers refer to experiments; $S$ and $L$ refer to (small and large) regions in Experiment 2; fast and slow refer to the speeds in Experiment 4. 
ered, where the latter is predicted by the static twodimensional geometric analysis shown in Figure 1.

\section{Summary of Effects on Quadratic Parameters}

Figure 8 summarizes the analysis of quadratic parameters by showing the values of peak location, curvature, and peak height as a function of probe diameter for each of the conditions and experiments presented here. Data points from the same experiment and condition are connected by a line, and all the conditions from an experiment have a common graphic symbol for that line. The introduction notes that it is possible to compare the values of curvature and peak location between conditions even from different experiments; however, under the assumptions and normalization process here, peak height can be compared only across conditions from the same experiment. Other data are available from previous studies using this paradigm (Klatzky \& Lederman, 1999; Lederman et al., 2000; Lederman et al., 1999), and we include them in the discussion of summary trends.

Peak location. The location of the peak of the quadratic has consistently been found to increase (i.e., occur at a sparser interelement spacing) as probe diameter increases (Klatzky \& Lederman, 1999; Experiments 1-4 here). Peak location has also been found to increase with increases in exploration speed (Lederman et al., 1999; Experiment 4). Finally, the zero-moment probe produced a higher peak location than did the stylus when the probe diameter was small ( 2 or $5 \mathrm{~mm}$ ).

Figure 8 shows the effects of probe diameter on peak location (interelement spacing value) across the present experiments. There are clearly two groups of functions, one for each type of plate. All the functions show that probe location moves to a wider spacing value with increasing probe diameter. The location is at a greater spacing value, however, for the plates used in Experiments 3 and 4 than for those used in Experiments 1 and 2.

These results implicate both geometric and exploratory factors in the location of the peak. The effects of probe diameter and plate type are to be expected from the analysis of the drop point in Figure 1, in that the probe tip will drop to the substrate at larger spacing values, as the probe diameter increases and the height of the elements increases. The effect of speed on the location of the peak is consistent with the idea that it will take a wider spacing value to trap the probe tip, since it moves more quickly. The difference in peak location between the zero-moment probe and the stylus may reflect differences in exploratory speed as well; this cannot be determined from the present results, since speed was self-controlled by the subject.

Curvature. Curvature measures the sensitivity of the roughness percept to variations in spacing, independently of the particular spacing values that were used in an experiment. Curvature magnitude has consistently been found to decrease as speed increased (Experiment 4; Lederman et al., 1999). Probe type also affected curvature, such that the zero-moment probe produced greater curvature magnitude than did the stylus when the probe diameter was small ( 2 or $5 \mathrm{~mm}$ ).
Effects of probe diameter on curvature have been inconsistent: Curvature magnitude was found here to be less for the 2-mm probe (Experiments 1 and 2) than for the 5- and 8-mm probes. In Klatzky and Lederman's (1999) data, however, the small probe produced greater curvature than did the large, and the present Experiments 3 and 4 failed to obtain a significant effect of diameter on curvature. Examination of Figure 8 suggests, however, that the failure to find effects of probe diameter on curvature magnitude in Experiments 3 and 4 may be due to the small diameters used. Across the overall range, there is an overall trend toward increasing curvature with increasing diameter. It is worth noting that despite the difference in probes and plates, the curvature values for the small probes in Experiments 3 and 4 (1- to 3-mm diameter) were similar, on average, to the values for the 2-mm probes in Experiments 1 and 2. This contrasts with the clear difference in the peak location effects for the two sets of plates.

At present, then, it appears that the sensitivity of roughness to variations in spacing, as measured by the magnitude of curvature, depends both on exploratory parameters and on the geometric relation between the probe and the textured surface. Exploratory parameters include speed and probe type-zero moment versus stylus. Faster speeds may produce noise that reduces the perceived difference between spacings. The zero-moment probe may produce greater sensitivity because it transmits vibrations directly to the finger pads.

Peak height. The peak height indicates the maximum roughness that is felt. It tended to decrease as probe diameter increased (nonsignificant trend in Experiment 1; significant in Experiments 2-4). Figure 8 shows these effects. Note that in this figure, it is possible to compare peak heights within an experiment (indicated by lines having a common graphic symbol), but not across experiments, owing to the magnitude estimation procedure and resulting normalization that is required.

Peak height has also been found to increase as speed increases (Lederman et al., 1999; Experiment 4). The effects of speed are complex, by the assumptions in Figure 2. Peak roughness could be related to the transition between frequency and/or amplitude effects on roughness near the drop point (see the bottom panels of Figure 2). Evidence that amplitude is a factor comes from Lederman et al. (2000), who found an increase in peak height when increased force was applied normal to the surface during exploration.

\section{Analysis of Probe/Plate Geometry}

We used the geometric analysis of the drop point, described in the introduction and Figure 1, to determine whether the peaks of the quadratic occurred in a systematic relation to the average spacing where the probe could just fall between the raised elements. Recall that this analysis uses a simplified static, two-dimensional model of the probe/plate interaction.

Experiments 1 and 2. Equation 1 was used to solve for the value of interelement spacing $(s)$ such that the 
probe achieves full penetration (i.e., $d=h$ ), given a probe of a particular radius $(r)$. This geometrically derived critical value of spacing was found to be $1.20,1.96$, and $2.50 \mathrm{~mm}$, for probes with diameters of 2,5 , and $8 \mathrm{~mm}$. In comparison, the average spacing at which the quadratic function for the zero-moment probe was found to peak, when log magnitude was fit to the HVD spacing value, was $1.08,1.60$, and $1.77 \mathrm{~mm}$, for the three probes, respectively. Thus, the peaks were located at $90 \%$ (2-mm probe), $82 \%$ (5-mm probe), and $71 \%$ ( $8-\mathrm{mm}$ probe) of the predicted critical spacing at which the probe falls between elements on average. (The comparable values for HV spacing were $0.77,1.14$, and $1.28 \mathrm{~mm}$, or about $60 \%$ of the critical spacing.) On the whole, this analysis suggests that for the zero-moment probe, peak roughness is obtained when the interelement spacing is below the critical value at which the probe tip fully penetrates between elements and falls to the bottom of the plate.

When the same analysis was done for the stylus probe, the disparity between the peak location and the critical spacing was greater: The roughness functions (HVD spacing) peaked at locations corresponding to $45 \%$ (2-mm probe), $67 \%$ (5-mm probe), and $70 \%$ ( $8-\mathrm{mm}$ probe) of the critical spacing value at which the probe would be expected to drop between elements.

Experiments 3 and 4. For the truncated-cone-shaped elements of Experiments 3 and 4, the geometric analysis in Figure 1B (hence, Equation 1) applies to the 3-mm probe, and the analysis in Figure $1 \mathrm{C}$ applies to the 1- and $2-\mathrm{mm}$ probes. We again solved for the value of interelement spacing $(s)$ such that the probe achieves full penetration (i.e., $d=$ height of cone), given a probe of a particular radius $(r)$. This geometrically derived critical value of spacing was found to be $1.16,1.72$, and $2.23 \mathrm{~mm}$ for probes with diameters of 1, 2, and $3 \mathrm{~mm}$.

Experiment 4 demonstrates clearly that static drop point geometry alone does not control the peak location in the magnitude estimation function, since the empirically obtained values were found to depend substantially on speed. This is in keeping with the analysis of speed effects in the introduction. The observed peaks for HVD spacing with the slowest speed (Experiment 4, slow) are $1.31 \mathrm{~mm}$ for the $1-\mathrm{mm}$ probe and $2.50 \mathrm{~mm}$ for the $3-\mathrm{mm}$ probe, both slightly higher than the drop point. In contrast, the empirically obtained values for the cylindrical elements of Experiments 1 and 2 were below the drop point. Although speed may be a factor, this discrepancy between the relation of the empirical to the geometrical critical values may reflect the very different shapes of the elements used-cylinders in Experiments 1 and 2 versus truncated cones in Experiments 3 and 4.

On the whole, the geometric analysis suggests that the relation between the drop point and the spacing at which the magnitude estimation function peaks depends on the nature of the probe, the plates, and exploratory factors. In future work, we intend to use plates with nonjittered elements and linear gratings, since the present predictions, which are based on average spacing values, may be thrown off by idiosyncratic aspects of the stimuli-especially when they are explored over a small area, as with the zero-moment probe of Experiments 1 and 2.

\section{Conclusions}

The present experiments expand our documentation of the effects of geometric and exploratory factors on roughness perception through a rigid probe, as recently summarized by Lederman and Klatzky (2001) and by Klatzky and Lederman (2002). Both types of factors appear to be critical, and multiple variables must be taken into account in order to account for the process. We have consistently confirmed the quadratic relation between log roughness magnitude and log interelement spacing in a textured plate. This holds for a variety of probes and plates and for various patterns of exploration that affect the loci of skin contacts, as well as the amplitude and frequency, of vibration. The present data, together with previous findings, show the importance of the probe tip diameter in relation to interelement spacing, the height of textured elements, the speed of exploration, and to a lesser extent, the type of probe.

Collectively, the data support our principal assumptionnamely, that a critical transition in roughness perception with a probe occurs at the drop point, the spacing value at which the probe makes a transition from riding above the elements to riding on the substrate. The theoretical analyses we have presented indicate that the drop point should move to higher spacing with increasing speed or probe tip diameter, causing a shift in the peak of the magnitude estimation function along the spacing axis. In addition, under the assumption that the roughness/frequency relation reverses near the drop point, a reversal of speed effects near the peak magnitude would be predicted. These patterns were observed. The present analyses, however, are but preliminaries to a detailed model of the probe/plate interaction, which is needed in order to account for geometric and exploratory effects.

Research on this topic is useful not only for a basic understanding of haptic perception, but also for applications in which a surface must be felt via a rigid interface. This is the case with teleoperator and virtual environment systems that display cutaneous and haptic feedback to the hand of a human operator, to provide feedback about the device that the operator is controlling. Vibratory cues have proven useful for simulating such events as surface contact and conveying the point of puncture (Kontarinis \& Howe, 1995). General domains of application include telesurgery, commerce using the Internet, and remote surface inspection. By modeling roughness perception through a probe, we hope to facilitate the development of these and other applications using vibrations as the source of texture perception.

\section{REFERENCES}

Birznieks, I., Jenmalm, P., Goodwin, A. W., \& Johansson, R. S. (2001). Encoding of direction of fingertip forces by human tactile afferents. Journal of Neuroscience, 21, 8222-8237. 
Connor, C. E., \& Johnson, K. O. (1992). Neural coding of tactile texture: Comparison of spatial and temporal mechanisms for roughness perception. Journal of Neuroscience, 12, 3414-3426.

FranzÉn, O. (1966). On summation: A psychophysical study of the tactual sense. In Quarterly progress and status report, Speech Transmission Laboratory, Royal Institute of Technology (pp. 14-25). Stockholm: Kungliga Tekniska Högskolan.

Hollins, M., Bensmaia, S., \& Risner, R. (1998). The duplex theory of tactile texture perception. In Proceedings of the 14th Annual Meeting of the International Society for Psychophysics (pp. 115-120).

Hollins, M., \& Risner, S. R. (2000). Evidence for the duplex theory of tactile texture perception. Perception \& Psychophysics, 62, 695705.

JohnSON, K.O., \& HsiaO, S. S. (1994). Evaluation of the relative role of slowly and rapidly adapting afferent fibers in roughness perception. Canadian Journal of Physiology \& Pharmacology, 72, 488-497.

KATZ, D. (1989). The world of touch (L. Krueger, Trans.). Hillsdale, NJ: Erlbaum. (Original work published 1925)

KLATZKy, R. L., \& Lederman, S. J. (1999). Tactile roughness perception with a rigid link interposed between skin and surface. Perception \& Psychophysics, 61, 591-607.

Klatzky, R. L., \& Lederman, S. J. (2002). Perceiving texture through a probe. In M. L. McLaughlin, J. P. Hespanha, \& G. S. Sukhatme (Eds.), Touch in virtual environments (pp. 180-193). Upper Saddle River, NJ: Prentice-Hall.

Kontarinis, D. A., \& Howe, R. D. (1995). Tactile display of vibratory information in teleoperation and virtual environments. Presence, $\mathbf{4}$, 387-402.

KudoH, N. (1988). Tactile perception of textured surfaces: Effects of temporal frequency on perceived roughness by passive touch. Tohoku Psychologica Folia, 47, 21-28.

LaMotte, R. H., \& Srinivasan, M. A. (1991). Surface microgeometry: Tactile perception and neural encoding. In O. Franzén \& J. Westman (Eds.), Information processing in the somatosensory system (pp. 4958). New York: Macmillan.

Lederman, S. J. (1974). Tactile roughness of grooved surfaces: The touching process and effects of macro- and microsurface structure. Perception \& Psychophysics, 16, 385-395.

Lederman, S. J. (1983). Tactual roughness perception: Spatial and temporal determinants. Canadian Journal of Psychology, 37, 498511.

Lederman, S. J., \& KLAtzky, R. L. (2001). Feeling surfaces and objects remotely. In R. Nelson (Ed.), The somatosensory system: Deci- phering the brain's own body image (pp. 103-120). Boca Raton, FL: CRC Press.

Lederman, S. J., Klatzky, R. L., Hamilton, C. [L.], \& Grindley, M. (2000). Perceiving surface roughness through a probe: Effects of applied force and probe diameter. Proceedings of the ASME Dynamic Systems \& Control Division, 69, 1065-1071.

Lederman, S. J., Klatzky, R. L., Hamilton, C. L., \& Ramsay, G. I. (1999). Perceiving roughness via a rigid stylus: Psychophysical effects of exploration speed and mode of touch. Haptics-e: The Electronic Journal of Haptics Research, 1, (1, October 8). www.haptics-e.org. Lederman, S. J., Loomis, J. M., \& Williams, D. A. (1982). The role of vibration in the tactual perception of roughness. Perception \& Psychophysics, 32, 109-116.

Lederman, S. J., \& TAYlor, M. M. (1972). Fingertip force, surface geometry, and the perception of roughness by active touch. Perception \& Psychophysics, 12, 401-408.

Sherrick, C. E. (1960). Observations relating to some common psychophysical functions as applied to the skin. In G. R. Hawkes (Ed.), Symposium on cutaneous sensitivity (pp. 147-158). Ft. Knox, KY: U. S. Army Medical Research Laboratory.

Smith, A. M., Chapman, C. E., Deslandes, M., Langlais, J.-S., \& ThibodeaU, M.-P. (2002). Role of friction and tangential force variation in the subjective scaling of tactile roughness. Experimental Brain Research, 144, 211-223.

Stevens, S. S. (1957). On the psychophysical law. Psychological Review, 64, 153-181.

TAYlor, M. M., \& Lederman, S. J. (1975). Tactile roughness of grooved surfaces: A model and the effect of friction. Perception \& Psychophysics, 17, 23-36.

Verrillo, R. T., Fraioli, A. J., \& Smith, R. L. (1969). Sensation magnitude of vibrotactile stimuli. Perception \& Psychophysics, 6, 366372.

Yoshioka, T., Gibb, B., Dorsch, A. K., Hsiao, S. S., \& Johnson, K. O. (2001). Neural coding mechanisms underlying perceived roughness of finely textured surfaces. Journal of Neuroscience, 21, 6905-6916.

\section{NOTE}

1. A formal proof can be requested from the first author.

(Manuscript received December 31, 2001; revision accepted for publication October 10, 2002.) 